\title{
New Vegetable Variety List 23
}

\author{
Compiled by the Garden Seed Research Committee \\ American Seed Trade Association
}

\author{
Edited By E.C. Tigchelaar \\ Department of Horticulture, Purdue University, West Lafayette, IN 47906
}

\begin{abstract}
Twenty-two prior lists of new vegetable varieties introduced since 1936 have been published by the American Society for Horticultural Science. This 23rd listing includes 376 varieties of vegetable crops released by public and private vegetable breeding programs.

The following abbreviations are used: exp. desig. - experimental designation; orig. - originated at or by; intr. - introduced by; par. - parentage or parents used; char. - outstanding characteristics; res.-_resistant to; tol.- tolerant to; sim.-similar to or resembles; sel.—selection or selected; adapt.—adaptcd to or adaptation.
\end{abstract}

\section{ASPARAGUS}

Brock Imperial 84 -1984-Orig. and Intr. by Arco Seed Co.

\section{BASIL}

Green Bouquet -1983-Orig. and Intr. by W. Atlee Burpee Co. Open Poll. Char. Dwarf bush plants with small dark-green leaves. Attractive as border. Sim. Picollo. Adapt. Wide.

\section{BEANS, GARDEN}

Atlantic (Exp. desig. XPB 148) - Orig. and intr. by Asgrow Seed Co. Open poll. Char. Very long pods; colored seed coat. Res. BCMV. Sim. Gatorgreen. Adapt. Eastern U. S.; other bean areas. PVP application pending.

Blazer (Exp. desig. GP 826414)-1986-Orig. and intr. by Rogers Bros. Seed Co. Open poll. Char. Erect bush and medium sieve size, green pods $51 \frac{1}{2}$ " to 6 "long. Res. Common Mosaic and NY 15 strain, tol. to some Rust strains. Sim. BBL 47. Adapt. Wide. PVP application No. 8500094.

Bronco (Exp. desig. XPB 172) -1986-Orig. and intr. by Asgrow Seed Co. Open poll. Char. Fresh-market bean with straight, slender, dark, shiny pods. Res. BCMV. Sim. Strike. Adapt. All bean areas. PVP application pending.

Citation (Exp. desig. XPB 169) -1986-Orig. and intr. by Asgrow Seed Co. Open poll. Char. Slender podded processing bean. Res. BCMV. Sim. Eagle. Adapt. All bean areas. PVP application pending.

Cutlass (Exp. desig. MOX8036)-1985-Orig. and intr. by Harris Moran Seed Co. Open poll. Char. Bush Kentucky Wonder type. Pod similar to Greencrop, but less fibrous and persistent green color. Res. BCMV, NY 15 strains BMV. Sim. Greencrop. Adapt. So. California, Arizona, Bush Kent. Wonder areas. PVP application pending.

Eureka (Exp. desig. XPB 167)-1986-Orig. and intr. by Asgrow Seed Co. Open poll. Char. Small-sieve wax garden bean. Res. BCMV. Sim. Goldrush. Adapt. All wax bean areas. PVP application pending.

Gipsy (Exp. desig. XPB 4017)-1987-Orig. and intr. by Asgrow Seed Co. Open poll. Char. Borlotto bush type with improved red color. Sim. Taylor's Horticultural. Adapt. Italy. PVP application pending.
Hystyle (Exp. desig. MOX8033)-1985-Orig. and intr. by Harris Moran Seed Co. Open poll. Char. Processor variety with very erect habit for high-density harvest. Persistent green color. Fleshy pods. Dark pods $51 / 4$ " to $61 / 2$ ", resists lodging. Res. BCMV, NY 15 Strains BMV, Curly Top virus, tol. Rust. Sim. Bush Blue Lake 47. Adapt. All bean areas. PVP application pending.

Javelin (Exp. desig. FM 1555)-1985 -Orig. and intr. by FerryMorse Seed Co. Open. poll. Char. White-seeded fresh-market/processor type. Slim, very attractive, uniform round pods. Res. BV-1, BV-1a Common Bean Mosaic Virus. Sim. Sprite. Adapt. Florida, Atlantic Seaboard, New York, Wisconsin, California.

Latemar (Exp. desig. XPB 142)- 1985-Orig. and intr. by Asgrow Seed Co. Open poll. Char. Dark, shiny-podded, slender, bush bean with colored seedcoat. Res. BCMV. Sim. Slankette. Adapt. All bean areas. PVP Certificate No. 8400021.

Laureat (Exp. desig. XPB 158) - Orig. and intr. by Asgrow Seed Co. Open poll. Char. Small-podded wholepack type with good yield and quality. Res. BCMV (I gene), Anthracnose (ARE), Halo Blight (Mod. Res.). Sim. Gitana. Adapt. All bean areas. PVP application pending.

Madrigal (Exp. desig. XPB 128) -1984-Orig. and intr. by Asgrow Seed Co. Open poll. Char. Green-seeded flageolet bean with good set height, growth habit, and yield. Res. BCMV. Sim. Verbel. Adapt. France. PVP application pending.

Mikado Orig. and intr. by Sunseeds. Open poll. char. Four-sieve bush green bean, with dark-green, glossy, straight, smooth pods on an upright bush. Res. BCMV. Sim. Slenderwhite. Adapt. All bean areas. PVP application pending.

Minia -1985 or 1986-Orig. and intr. by Asgrow Seed Co. Open poll. Char. Bolotto bean with blue (violet) coloring and determinate bush plant type. Sim. Blue Borlotti. Adapt. Italy. PVP application pending.

Podsquad (Exp. desig. XPB 171) -1986-Orig. and intr. by Asgrow Seed Co. Open. poll. Char. High-yielding fresh-market bean with long, slender pods. Res. BCMV. Sim. Gatorgreen. Adapt. All bean areas. PVP application pending.

Sentry (Exp. desig. XPB 170)-1986-Orig. and intr. by Asgrow Seed Co. Open poll. Char. Small-sieve processing bean; low fiber and high yield. Res. BCMV. Sim. Strike. Adapt. All bean areas. PVP application pending.

Sprout (Exp. desig. XPB 189)-1986-Orig. and intr. by Asgrow Seed Co. Open poll. Char. Fresh-market garden bean with good germination characteristics. Res. BCMV. Sim. Sprite. Adapt. All bean areas. PVP application pending.

Tess (Exp. desig. XPB 161) -1985-Orig. and intr. by Asgrow Seed Co. Open poll. Char. Small-podded wholepack type with good yield and quality. Res. BCMV (I gene), Anthracnose (ARE), Halo Blight (Mod. Res.). Sim. Gitana. Adapt. All bean areas. PVP application pending.

Thrive (Exp. desig. XPB 195)-1987-Orig. and intr. by Asgrow Seed Co. Open poll. Char. Full-sieve processing garden bean with good germination characteristics. Res. BCMV. Sim. Bush Blue Lake 47. Adapt. All bean areas. PVP application pending.

Vista (Exp. desig. XPB 190)-1987-Orig. and intr. by Asgrow Seed Co. Open poll. Char. Dark-green, high-quality garden bean, for processing or fresh market. Good germination characteristics. Res. BCMV. Sim. Labrador. Adapt. All bean areas. PVP application pending. 
Volare (Exp. desig. XPB 154)-1985-Orig. and intr. by Asgrow Seed Co. Open poll. Char. Bush Romano type with improved standability; colored seed. Res. BCMV. Sim. Gina. Adapt. U. S., Italy, Spain, U.K.

\section{BEET}

Red Ace (Exp. desig. 4292)-1981-Orig. and intr. by Alf Christianson Seed Co. F1 Hybrid. Par. Female, 375A. Char. Fastgrowing early maturing hybrid for home garden, market, and pro cessor. Smooth root with good color and resists zoning. High sugar for good taste. Bolt-resistant. Strong petioles for erect growth. Bright green leaf. Res. Tol. Cercospora Leaf Spot. Sim. Detroit Dark Red. Adapt. Wide.

Detroit Supreme -1984-Orig. and intr. by Alf Christianson Co. Open poll. Char. Root color very even and dark. Very smooth. Good dark outside color. Shape round to somewhat high-round. Leaves: Medium length with small root attachment. Long petioles. Res. Downy Mildew, Tol. Peronospora farinosa F. sp. Betae. Sim. Detroit Dark Red. Adapt. Wide.

\section{BROCCOLI}

Apex - Orig. and intr. by Sunseeds. F1 Hybrid. Char. Early, short, medium bead size. Sim. Futura. Adapt. Wide

Baccus (Exp. desig. XPH 5003)-1986-Orig. and intr. by Asgrow Seed Co. F1 Hybrid. Char. Early, heat-tolerant hybrid for Midwestern U.S. Sim. Packman and Galaxy. Adapt. Great Lakes, Eastern Seaboard.

Bonanza -1982-Orig. and intr. by Known-You Seed Co., Vendor: W. Atlee Burpee Co. F1 Hybrid. Char. Large, tight, central heads and excellent production of side shoots after central head is cut. Sim. Premium Crop. Adapt. Wide.

Chancellor (Exp. desig. NVH 510) (\#90)-1983-Orig. and intr. by Northrup King Co. F1 Hybrid. Char. Medium-dark green; heads $41 / 2$ " to $51 / 2$ " with medium extension, fine beads, and a medium dome. Excellent fresh-market hybrid with good holding ability. Sim. Hybrid Emperor. Adapt. Texas and desert areas.

Commodore (Exp. desig. GSV 2820) -1983-Orig. and intr. by Northrup King Co. F1 Hybrid. Char. Medium-late hybrid, wellshaped domed heads, small to medium bead, thick stems, heads are at top of wrappers. Sim. Hybrid Shogun. Adapt. Early season plantings.

Galaxy (Exp. desig. XPH 5166)-1984-Orig. and intr. by Asgrow Seed Co. F1 Hybrid. Char. Very high-yielding, early hybrid. Sim. Premium Crop. Adapt. Coastal areas and fall planting in Midwestern U.S.

Vantage - Orig. and intr. by Sunseeds. F1 Hybrid. Char. Midseason, medium height, heads produce above foliage for ease of harvest. Sim. Emperor. Adapt. Wide.

\section{CABBAGE}

Dynasty (Exp. desig. XPH 1104)-1985-Orig. and intr. by Asgrow Seed Co. F1 Hybrid. Adapt. Rio Grande Valley and Mexico. Res. Yellows, Tol. Downy Mildew. Sim. Market Prize.

Early Curly (Exp. desig. NO 8010)-1985-Orig. and intr. by W. Atlee Burpee Co. F1 Hybrid. Char. Early savoy type with heavily crinkled leaves, small core, and sweet flavor. Sim. Salarite. Adapt. Wide.

Genesis (Exp. desig. ARCO 206)-1987-Orig. and intr. by Arco Seed Co. F1 Hybrid. Char. Midseason fresh-market type. Greenbluegreen color. Medium to large frame with good wrappers. Round heads with solid interiors and medium-short cores. Res. Fusarium Yellows, Tol. Xanthomonas Black Rot. Sim. Bravo. Adapt. Wide.

Gloria (Exp. desig. XPH 1058)-1984-Orig. and intr. by Asgrow Mexicana. F1 Hybrid. Char. Mid-early maturity, round-head344 ed shipper. Uniform, holds well in field. Sim. Express (Asgrow) or Viking (Agway). Adapt. Mexico only.

Ocala - Orig. and intr. by Sunseeds. F1 Hybrid. Char. Midseason, dual purpose for fresh market or processor, good holding quality. Res. Fusariurn Yellows, Black Rot. Sim. Rio Verde. Adapt. Wide.

Pennant (Exp. desig. NVH 672)-1985-Orig. and intr. by Northrup King Co. F1 Hybrid. Char. Fresh-market type. Medium frame size, blue green in color, good wrapper leaves. Healthy appearance under a wide range of climatic conditions. Matures a week earlier than Rio Verde. Holding ability excellent. Sim. Rio Verde. Adapt. Wide.

Show Boat (Exp. desig. HYB MOX 9078)-1985-Orig. and intr. by Harris Moran Seed Co. F1 Hybrid. Char. Mid-season fresh market hybrid, early maturing with good holding ability. Res. Black Rot, Yellows. Sim. Bravo. Adapt. North Carolina, South Carolina.

Two Seasons Hybrid -1982-Orig. and intr. by W. Atlee Burpee Co. F1 Hybrid. Char. Chinese Cabbage with short broad heads. Very compact. Does well as either spring or fall crop. Res. Heat, soft rot, and bolting. Sim. Burpee Hybrid. Adapt. Wide.

\section{CA R R O T}

Apache -1985-Orig. and intr. by Arco Seed Co. F1 Hybrid. Char. Strong tops; good early vigor; excellent color and smoothness; good root weight into tip; for bunching or cello. Res. Tol. Alternaria, Cercospera. Sim. Imperator type. Adapt. Wide.

Atlantis [Exp. desig. $(4367 \times 3640) \times 3180]-1987$-Orig. and intr. by Dept. of Hort., Univ. of Wisconsin, USDA/ARS. F1 Hybrid. Char. Early crop production. Res. Tol.Alternaria. Sim. Imperator 58. Adapt. All carrot-producing areas.

Beta 111 -1987-Orig. and intr. by Dept. of Hort., Univ. of Wisconsin, USDA/ARS. Open poll. Char. Good flavor, color, and high carotene. Res. Alternaria. Sim. Imperator 58. Adapt. All carrot-producing areas.

Brasilia (Exp. desig. XP 5069)-1984-Orig. and intr. by EMBRAPA. Vendor: Asgrow Seed Co. Open poll. Char. Uniform Nantes type. Sim. Kuronan. Adapt. Central America/Northern Brazil.

Carolong [Exp. desig. $(6439 \mathrm{~S} \times 6274 \mathrm{M}) \mathrm{X}$ ]-1988-Orig. and intr. by USDA/ARS. F1 Hybrid. Char. long, cylindrical type adapted to European market. Res. Alternaria. Sim. Long Nantes. Adapt. Nantes areas worldwide.

Caropak (Exp. desig. XPH 569)-1985-Orig. and intr. by Asgrow Seed Co. F1 Hybrid. Char. Widely-adapted US mechanical harvest hybrid with excellent nutritional and visual quality. Res. Cercospora, Tol. Alternaria. Sim. Chancellor. Adapt. U.S., Canada.

Cello King (Exp. desig. NVH 1010)-1984-Orig. and intr. by Northrup King Co. F1 Hybrid. Char. Imperator class for fresh market or slicing. Roots are 9" to 10" in length and 11/4" to $11 / 2$ " in diameter. Good exterior and interior color and uniformity. Strong foliage attachment. Top 18" to 21" long. Resists bolting. Res. Alternaria leaf blight. Sim. Woodland, Fanci Pak, Sierra, Sundance. Adapt. All fresh-market growing areas.

Centennial (Exp. desig. XPH 982)-1986-Orig. and intr. by Asgrow Seed Co. F1 Hybrid. Char. Excellent interior and exterior quality for slicing for freezing and canning. Res. Alternaria and Cercospora. Sim. Semi-Nantes. Adapt. Wisconsin, Great Lakes.

Dagger 78 -1978 - Orig. and intr. by Arco Seed Co. F1 Hybrid. Char. High color, smooth, with good tip fill. Adapt. All carrot-producing areas.

Discovery (Exp. desig. NVH 1007)-1984-Orig. and intr. by Northrup King Co. Char. Imperator style for fresh market or slicing. Roots slightly tapered $91 / 2$ " to $101 / 4$ " long and $11 / 4$ " diameter. Good exterior and interior color and uniformity. Dark-green, strong tops 20" to 22" long. Resists bolting. Res. Alternaria leaf blight. Sim. Fanci Pak, Woodland, Sierra, and Sundance. Adapt. All carrot-producing areas.

Eagle (Exp. desig. ARCO 209)-1987-Orig. and intr. by Arco Seed Co. F1 Hybrid. Char. Slicer type (9" to 10' length) with good color and less-green shoulder than cur-rent standards. Sim. Heavy HortScIEnce, Vol. 26(4), APrIL 1991 
Nantes and Scarlet Nantes. Adapt. Organic soils in Wisconsin, Indiana, Michigan, Ontario, Quebec.

Falcon -1987-Orig. and intr. by Arco Seed Co. Open poll. Char. Slicer type 8" to 9" in length with blunt tip. Sim. Heavy Nantes. Adapt. Michigan, Wisconsin, Ontario, Quebec.

Gallena (Exp. desig. XPH 5120)-1985-Orig. and intr. by Asgrow Seed Co. F1 Hybrid. Char. Early, long Nantes type. Sim. Asgrow Nantes. Adapt. Northern Europe, U.S.

Huron (Exp. desig. ARCO 178)-1987-Orig. and intr. by Arco Seed Co. F1 Hybrid. Char. Good color, smooth, good tip wt., 9" to 11". Sim. Imperator class carrots. Adapt. Michigan, Wisconsin, Ontario, Quebec, Central California, Colorado, Washington.

Javelin 80 -1980-Orig. and intr. by Arco Seed Co. F1 Hybrid. Char. Long, slender, straight roots with slight taper. Sim. Irnperator 58. Adapt. California.

24 Karat (Exp. desig. FMX 166)-1985-Orig. and intr. by Ferry-Morse Seed Co. F1 Hybrid. Par. FM inbreds. Char. Long Slender Gold Pak type with smooth cylindrical roots. Excellent crack resistance, very good color, no premature bolting tendency. Res. Powdery Mildew. Sim. Trophy. Adapt. California, Midwest.

Kuronan (Exp. desig. XP 513C)-1984-Orig. and intr. by EMBRAPA. Vendor: Asgrow Seed Co. Open poll. Char. high-quality selection of Kuroda. Uniform interior for tropical summer produc tion. Res. Alternaria . Sim. Kuroda. Adapt. Tropical summer production.

Mark II (Exp. desig. FMX 105) -1985-Orig. and intr. by Ferry-Morse Seed Co. F1 Hybrid. Par. FM inbreds. Char. Chantenay type with smooth blunt-tip, round-shouldered roots, with uniform interiors. Good bolting tolerance and crack resistance. Sim. Chantenay types. Adapt. Wisconsin, Canada, California.

Savory (Exp. desig. 403-3)-1986-Orig. and intr. by Dept. of Hort., Univ. of Wisconsin, USDA/ARS. Vendor: Arco Seed Co. F1 Hybrid. Par. $(8080 \mathrm{~S} \times 2302) \times 2566$. Char. High carotene and excellent flavor. Res. Tol. Alternaria leaf blight. Sim. Orlando Gold. Adapt. U.S.

Seminole -1985-Orig. and intr. by Arco Seed Co. F1 Hybrid. Char. Early; strong tops; good color; root fills well to tip. Res. Tol. Alternaria. Sim. Imperator type. Adapt. Most shipping carrot production areas.

Six Pence (Exp. desig. Exp. Hyb. 2914)-1985-Orig. and intr. by Harris Moran Seed Co. F1 Hybrid. Char. Hicolor Imperator type, with long semi-cylindrical roots 9" to 10 ". Avg. root diam. $11 / 4$ ", tops 18 " to 20". Sim. Imperator 58. Adapt. Fresh-market areas.

Sprinter (Exp. desig. Exp. Hyb. 2101)-1985-Orig. and intr. by Harris Moran Seed Co. F1 Hybrid. Char. Imperator 58 type, good vigor, long cylindrical roots (8" to 9") avg. diam. $11 / 4$ ", tops 18 " to 20". Sim. Imperator 58. Adapt. Fresh-market areas.

Toudo Hybrid -1985-Orig. and intr. by W. Atlee Burpee Co. F1 Hybrid. Char. Nantes type-cylindrical stumped roots that resist splitting. Sim. Nantes. Adapt. Wide.

\section{CAULIFLOWER}

Candid Charm (Exp. desig. NVH 1505)-1983-Orig. and intr. by Northrup King Co. F1 Hybrid. Char. Uniform, vigorous, tightdomed head; later-maturing than Snowball Y. Does not produce purplish color on head. Sim. Glacier and Snowball Y. Adapt. All cauliflower-producing areas.

Early Glacier (Exp. desig. NVH 1506)-1983-Orig. and intr. by Northrup King. Co. F1 Hybrid. Char. About 10 days earlier than Glacier and Snowball Y; tight, dome-shaped head; good vigor. Sim. Snowball Y and Glacier. Adapt. All cauliflower-producing areas.

Early Snowflake - Orig. and intr. by Dessert Seed Co. Open poll. Char. Early. Sim. Tropical types. Adapt. Warm regions.

Glacier (Exp. desig. NVH 1504)-1983-Orig. and intr. by Northrup King Co. F1 Hybrid. Char. uniform, tight, dome-shaped head; Snowball Y season. Sim. Snowball Y. Adapt. All cauliflowerproducing areas.

Igloo - Orig. and intr. by Sunseeds. Open poll. Char. Late-season type, excellent wrapper leaves. Sim. Snowball Y. Adapt. Winter growing areas.

HortSCIENCE, Vol. 26(4), APRIL 1991
Majestic (Exp. desig. NVH 1503)-1983-Orig. and intr. by Northrup King Co. F1 Hybrid. Char. Vigorous; heavy dome-shaped head; break slowly. Res. Tol. mildew. Sim. Snow Crown. Adapt. All cauliflower-producing areas.

Snowball T-1 - Orig. and intr. by Dessert Seed Co. Open poll. Sim. Snowball types. Adapt. Southern California desert.

Snowball T-2 -Orig. and intr. by Dessert Seed Co. Open poll. Sim. Snowball types. Adapt. Southern California desert.

Snowball T-3 - Orig. and intr. by Dessert Seed Co. Open poll. Sim. Snowball types. Adapt. Southern California desert.

Snowball T-4 -Orig. and intr. by Dessert Seed Co. Open poll. Sim. Snowball types.

\section{CUCUMBER}

Aurora (Exp. desig. ARCO 699)-1985-Orig. and intr. by Arco Seed Co. F1 Hybrid. Par. ARCO Seed parent $559 \times$ NCSU pollen parent (Clinton). Char. Indeterminate vine, uniform fruit shape, white spine, good fruit color, small seed cavity, late seed development, 1/d 3.0. Res. Scab, CMV, ALS, PM, DM, and Anthracnose . Sim. Calico. Adapt. Texas, Florida, North Carolina, Michigan.

Beauty -1983-Orig. and intr. by Hollar and Co., Inc. F1 Hybrid. Char. Gynoecious, parthenocarpic, designed for greenhouse use only. Fruit 13" long. Sim. Pepinex, Corona, Deleva.

Burpee Hybrid 11 -1984-Orig. and intr. by W. Atlee Burpee Co. F1 Hybrid. Char. Earlier and more productive than Burpee Hybrid; slightly longer fruits. Res. Mosaic and Downy Mildew. Sim. Burpee Hybrid. Adapt. Wide.

Burpeeana Hybrid II -1984-Orig. and intr. by W. Atlee Burpee Co. F1 Hybrid. Char. Gynoecious form of Burpeeana; earlier and more productive. Sim. Burpeeana Hybrid. Adapt. Wide.

Castlemaster (Exp. desig. CHY 2506)-1984-Orig. and intr. by Arco Seed Co. F1 Hybrid. Char. medium-early indeterminate slicer with uniform color. Good yield and femaleness. Res. Scab, CMV, PM, DM. Sim. Slicemaster. Adapt. Southern U.S., Mexico.

Castlepickle 183 -Orig. and intr. by Arco Seed Co. F1 Hybrid. Char. Intermediate pickling vine; Medium fruit length (L/D 2.8) and fruit color. Res. Scab, CMV, Verticillium. Adapt. Western California.

Castlepik (Exp. desig. CHY 2012)-1984-Orig. and intr. by Arco Seed Co. Char. Determinate, prolific, white spine pickling type; medium fruit color and length (L/D 2.9). Res. Scab, CMV, PM, DM. Adapt. Any pickle-producing area.

Castleroyal (Exp. desig. CHY 2013)-1985-Orig. and intr. by Arco Seed Co. F1 Hybrid. Char. Vigorous vine pickling type for multiple harvest. Good fruit length (L/D 3.0) and dark-green fruit color. White spines. Res. Scab, CMV, ALS, Anthracnose, PM, DM. Sim. Calypso. Adapt. Eastern U.S., Canada.

Comet A (Exp. desig. XPH 1472)-1985-Orig. and intr. by Asgrow Seed Co. F1 Hybrid. Char. Early slicer type; (L/D 4.0). Res. Cucumber scab. Sim. Marketmore 76. Adapt. Eastern and Midwestern U.S., Western Mexico, Texas.

Delta (Exp. desig. ARCO 769)-1986-Orig. and intr. by Arco Seed Co. F1 Hybrid. Char. Indeterminate pickling type; mediumgreen fruit color, good fruit length (L/D 3.0+) in wide range of environments. Res. Scab, CMV, ALS, Anthracnose, Powdery Mildew, Downy Mildew. Sim. Regal. Adapt. Midwest, East Coast, Carolinas.

Diyala (Exp. desig. XPH 1475) -1985-Orig. and intr. by Asgrow Seed Co. F1 Hybrid. Char. Cylindrical fruit medium-green color, slightly rough skin $(\mathrm{L} / \mathrm{D} \approx 3.6)$. Sim. Arabel type. Adapt. Middle East, protected culture.

Early Pride Hybrid -1983-Orig. and intr. by W. Atlee Burpee Co. F1 Hybrid. Char. Early gynoecious slicing cucumber with darkgreen color; produces over long season. Res. Tol. to Mosaic and Mildew. Sim. Triumph. Adapt. Wide.

Fancipak (Exp. desig. XPH 1369)-1985-Orig. and intr. by Asgrow Seed Co. F1 Hybrid. Char. Dark color, hand-harvest pickling type with low incidence of crooks and nubs. Res. Tol. to Anthracnose, DM, Scab, ALS, CMV, PM. Sim. Score. Adapt. Warm growing areas. 
Fremont (Exp. desig. $1983 \times$ Clinton)-1985-Orig. and intr. by USDA and Wisconsin AES. Vendor: Arco Seed Co. F1 Hybrid. Par. W1983G × Clinton. Char. White spine, LD 2.85, blocky fruit, good fruit color distribution, medium-size vine that remains healthy into season. Res. ALS, PM, CMV, and Scab. Sim. Calico. Adapt. Ohio, Michigan, Wisconsin, North Carolina.

Hybrid Aseel -Orig. and intr. by Sunseeds. F1 Hybrid. Char. Ability to set dark-green, shiny fruits without pollination and set an early crop of fruits. Res. Powdery Mildew. Sim. Hybrid Beit Alpha. Adapt. Middle Eastern areas; any area with Mediterranean climate.

Jade (Exp. desig. XPH 1367)-1984-Orig. and intr. by Asgrow Seed Co. F1 Hybrid. Char. Early Middle Eastern type for open field culture. L/D 3.7. Medium-green, thin shiny skin. Very productive. Res. Tol. CMV. Sim. Beit Alpha. Adapt. Middle East, where Beit Alpha types used.

Longreen -Orig. and intr. by Hollar and Co., Inc. F1 Hybrid. Char. Closely resembles Marketmore 76, but with hybrid color; fruit 9", uniformly dark green. Res. Downy and Powdery Mildew, scab. Adapt. Wide.

Manisa (Exp. desig. XPH 1421)-1985-Orig. and intr. by Asgrow Seed Co. F1 Hybrid. Char. An early Beit Alpha type for growth under polyethylene tunnels, where its resistance to both downy and powdery mildew is a distinct advantage. Res. Downy/ Powdery Mildews. Sim. Beit Alpha. Adapt. Middle East, E. Mediterranean.

Nadine (Exp. desig. XPH 1413) -1985-Orig. and intr. by Asgrow Seed Co. F1 Hybrid. Char. Smooth-skinned fruit with moderately dark color, $L / D \approx 3.6$; vines vigorous and highly female. Sim. Marbosan and Arabel. Adapt. Middle East, protected culture.

Olympian F1 (Exp. desig. ss138)-1985-Orig. and intr. by Hollar and Co., Inc. F1 Hybrid. Res. Scab, Angular Leaf Spot, Downy Mildew, Powdery Mildew.

Primepak (Exp. desig. XPH 1530)-1986-Orig. and intr. by Asgrow Seed Co. F1 Hybrid. Char. Long, dark color pickling hybrid. Sim. Regal. Adapt. Most pickle areas.

Rock -1983-Orig. and intr. by Hollar and Co., Inc. F1 Hybrid. Char. Parthenocarpic for plastic tunnel or greenhouse production. Fruit 12 to $15 \mathrm{~cm}$ Gynoecious. Sim. Arabelle, Marbosan.

Sweet Delight (Exp. desig. ARCO 8)-1983-Orig. and intr. by Arco Seed Co. F1 Hybrid. Char. White-spined burpless slicer type hybrid with long (10" to 12") fruit and thin skin. The vines are indeterminate. Res. Scab, CMV, PM. Sim. Sweet Slice, Sweet Success. Adapt. Home and truck gardens.

Tigris (Exp. desig. ARCO 760) -1985-Orig. and intr. by Arco Seed Co. F1 Hybrid. Char. White-spined Beit Alpha type with indeterminate vines, good color and fruit length. Mild flavor. Res. CMV. Adapt. Middle East, home gardens in U.S.

\section{EGGPLANT}

Early Prolific F-2 - Orig. and intr. by Dessert Seed Co. F2. Sim. Florida Market. Adapt. Wide.

\section{LEEK}

Gabilan (Exp. desig. 325.012)-1984-Orig. and intr. by Alf Christianson Seed. Co. Open poll. Char. Shaft very long and white. No bulbing. Leaves are dark green and upstanding. Fast growing. Sim. American Flag. Adapt. Areas with winter temperature -10C.

\section{LETTUCE}

Bix (Exp. desig. XP 5228)-1986-Orig. and intr. by Asgrow Seed Co. Open poll. Char. uniform, with small core. Sim. Salinas. Adapt. Coastal growing areas of California.

Cannon (Exp. desig. XP 993)-1986-Orig. and intr. by Asgrow Seed Co. Open poll. Char. For warmer-season production. Sim. Fairton. Adapt. Eastern U.S. lettuce production areas.
Classic (Exp. desig. XP 5171)-1986-Orig. and intr. by Asgrow Seed Co. Open poll. Char. uniform fall lettuce. Res. LMV. Sim. Empire. Adapt. California San Joaquin Valley fall production.

Fame (Exp. desig. XP 5172)-1986-Orig. and intr. by Asgrow Seed Co. Open poll. Char. Earlier-maturing than Empire. Res. LMV. Sim. Empire. Adapt. Imperial Valley of Californa; Yuma, Ariz.

Gemstone -1981-Orig. and intr. by Arco Seed Co. Open poll. Char. Dark-green Savoy loose-leaf lettuce.

Greenfield (Exp. desig. P-20, Exp. 320)-1985-Orig. and intr. by Harris Moran Seed Co. Open poll. Char. Early, uniform, concentrated harvest. Leaves do not curl in on top at maturity; more intense green color than PI Cos; good shipping quality. Res. Oshita Virus Disease, Tol. Corky Root. Sim. PI Cos. Adapt. California coastal valleys, Romaine growing areas. PVP application pending.

Green Towers (Exp. desig. P-20, Exp. 320)-1985-Orig. and intr. by Harris Moran Seed Co. Open poll. Char. Early, uniform, concentrated harvest, taller than PI Cos. Leaves do not curl in on top at maturity. More intense green color. Large attractive butt, good shipping ability. Res. Oshita Virus disease, Tol. to Corky Root. Sim. PI Cos. Adapt. California coastal valleys, Romaine growing areas. PVP application pending.

Little Leprechaun -1983-Orig. and intr. by Arco Seed Co. Open poll.

Rosa - 1981-Orig. and intr. by Arco Seed Co. Open poll. Char. Red head lettuce.

Rubra -1981-Orig. and intr. by Arco Seed Co. Char. Red Cos Lettuce.

Salad Bibb (Exp. desig. HXP 3550)-1985-Orig. and intr. by Harris Moran Seed Co. Open poll. Char. Summer Bibb-type lettuce with multiple disease resistance. Res. Lettuce Mosaic Virus, Broad Bean Wilt virus and Downy Mildew. Sim. Summer Bibb. Adapt. Northeastern, Midwestem, Southern U.S. PVP application pending.

Sweetie B.S. -1981-Orig. and intr. by Arco Seed Co. Char. Semi-Cos leaf lettuce.

\section{MUSKMELON}

All Star (Exp. desig. HXP 3587)—Orig. and intr. by Harris Moran Seed Co. F1 Hybrid. Char. Shipping muskmelon adapted to South and East; good quality and fruit size. Sim. Magnum 45. Adapt. Florida and Northeast.

Alwaha (Exp. desig. FMX 44)-1985-Orig. and intr. by FerryMorse Seed Co. F1 Hybrid. Char. A more completely netted Ananas type with large size, yellow-orange color, excellent taste and aroma. Vigorous vines with good cover. Sim. Ananas. Adapt. Central California Middle East.

Aragon (Exp. desig. XPH 5094)-1986-Orig. and intr. by Asgrow Seed Co. F1 Hybrid. Char. Western shipper type, round, sutureless, small seed cavity, even, close net; makes size under stress conditions. Sim. Laguna. Adapt. Southern Texas, Imperial Valley of California, Western Mexico.

Aurora (Exp. desig. AC-68-52.) - Orig. and intr. by Auburn Univ. Open poll. Par. AC-63-11 × PI 14047. Char. Large fruit, high quality. Res. Gummy Stem Blight Downy Mildew, Powdery Mildew. Sim. Planter's Jumbo. Adapt. Southern U.S. and similar climates.

Buccaneer (Exp. desig. NVH 877)-1983-Orig. and intr. by Northrup King Co. F1 Hybrid. Char. Fruit slightly oval 6" $\times 61 / 2$ ". Moderate sutures. Fine netting; thick, bright-orange flesh. Matures in $\approx 76$ days. Adapt. home garden-truck farm, short-distance type. Res. Powdery and Downy Mildew. Sim. Hybrid Supermarket. Adapt. Eastern U.S.

Castle Rock (Exp. desig. 5004)-1987-Orig. and intr. by Arco Seed Co. F1 Hybrid. Char. Res. Powdery Mildew. Sim. Topmark. Adapt. Western U.S.

Challenger (Exp. desig. NVH 882)-1983-Orig. and intr. by Northrup King Co. F1 Hybrid. Char. Sutureless, round-oval fruit, well-netted, firm flesh, small cavity. Midseason maturity, shipper type. Res. Powdery Mildew, races 1 and 2 and some Downy MilHortSCIEnCE, Vol. 26(4), AprIL 1991 
dew. Sim. Imperial 45, Top Mark, and Perlita types. Adapt. California, Arizona, Texas.

Columbia (Exp. desig. NVH 876)-1983-Orig. and intr. by Northrup King Co. F1 Hybrid, Char. Slightly oval fruit 5 " $\times 51 / 2$ ", moderate sutures. Fruit well-netted, medium-orange, thick flesh. Very good flavor. Maturity $\approx 76$ days. A home garden-truck farm type. Res. Powdery and Downy Mildew. Sim. Hybrid Supermarket. Adapt. All muskmelon-producing areas.

Conqueror (Exp. desig. NVH 875)-1983-Orig. and intr. by Northrup King Co. F1 Hybrid. Char. Slightly oval fruit 5 1/2" x 6". Fine netting and slightly sutured. Medium-orange, thick flesh. Maturity $\approx 76$ days for home garden and truck farm use. Res. Powdery and Downy Mildew. Sim. Hybrid Supermarket. Adapt. All muskmelon-producing areas.

Conquistador (Exp. desig. GSV 83-92)-1983-Orig. and intr. by Goldsmith Seeds, Inc., of Northrup King Co. F1 Hybrid. Char. Early maturing; fruit oval, deep-sutured, coarse-netted, averaging 3 $1 / 2$ to $4 \mathrm{lb}$. Sweet, firm, orange closed flesh. Sim. Early Dawn.

Cortez (Exp. desig. XPH 5098)-1985-Orig. and intr. by Asgrow Seed Co. F1 Hybrid. Char. An early, large-fruited, sutured melon, pink-fleshed, with small core. For areas where earliness and large size is needed. Sim. Planter's Jumbo. Adapt. Eastern U.S.

Earligold F1 (Exp. desig. EX-29-6A)-1985-Orig. and intr. by Hollar and Co., Inc. F1 Hybrid. Char. 5 days earlier than Goldstar. Sim. Jumbo-type melon. Adapt. Northern latitudes.

Earlimark F1 (Exp. desig. SS127) -1985-Orig. and intr. by Hollar and Co., Inc. F1 Hybrid. Char. Tol. to sulphur. Res. Powdery Mildew. Sim. Topmark. Adapt. Western U.S.

Early Eastern Shipper (Exp. desig. HYB 22545)-1987-Orig. and intr. by Arco Seed Co. F1 Hybrid. Char. Early PMR 45 type. Res. Powdery Mildew, Fusarium Wilt. Adapt. Northern U.S.

Explorer (Exp. desig. NVH 881)-1983-Orig. and intr. by Northrup King Co. F1 Hybrid. Char. Round oval fruit with wellnetted sutures. Firm, sweet, bright-colored flesh. Midseason maturity. Shipper type. Res. Powdery Mildew (races 1 and 2), Downy Mildew. Sim. Tamuvalde, Mainstream, Perlita. Adapt. Texas, Southeastern U.S.

Galo (Exp. desig. XPH 5183)-1985-Orig. and intr. by Asgrow Seed Co. F1 Hybrid. Char. Early, "Galia" class melon for shipping to distant markets. Sim. Galia. Adapt. Spain, Eastern Mediterranean.

Gold Master (Exp. desig. SHP)-1987-Orig. and intr. by Arco Seed Co. Open poll. Sim. Shipmaster. Adapt. Central California.

Golden Harvest (Exp. desig. HYB 30516)-1988-Orig. and intr. by Arco Seed Co. F1 Hybrid. Char. fresh-market muskmelon. Res. Fusarium, Powdery Mildew. Sim. Gold Star. Adapt. Northeastern U.S.; central California.

Golden Pride (Exp. desig. HYB 30436)-1988-Orig. and intr. by Arco Seed Co. F1 Hybrid. Char. disease-resistant Harper Hybrid. Res. Fusarium, Powdery Mildew (races 1 and 2). Sim. Harper Hybrid. Adapt. Northeast U.S.; central California.

Golden Shipper (Exp. desig. Hybrid 22515 )-1988-Orig. and intr. by Arco Seed Co F1 Hybrid. Char. Early shipper Res. Fusarium and Powdery Mildew. Adapt. Northeastern U. S.; central California.

Harvest Pride (Exp. desig. 5003)-1986-Orig. and intr. Arco Seed Co. F1 Hybrid. Char. Res. Powdery mildew. Sim. Topmark. Adapt. Southwestern U.S.

Honey Moon -1987-Orig. and intr. by Arco Seed Co. F1 Hybrid. Char. Disease-resistant Honeydew. Res. Powdery Mildew, Downy Mildew. Adapt. Texas.

Honeybush - Orig. and intr. by W. Atlee Burpee Co. Open poll. Char. Short internode- (bush-) type muskmelon bearing three to six melons ranging in size from $21 / 2$ to $3 \mathrm{lb}$. Thick, orange flesh with small seed cavity. Res. Fusarium Wilt. Sim. Honey Rock. Adapt. Wide.

Intrepid (Exp. desig. NVH 880)-1983-Orig. and intr. by Northrup King Co. F1 Hybrid. Char. No sutures, fully netted; excellent interior quality shipper type in Shipmaster and Tam Uvalde season. Res. Powdery Mildew (race 1) and some Downy Mildew strains.

Limelight Hybrid -1984-Orig. and intr. by W. Atlee Burpee HortSCIENCE, Vol. 26(4), APRIL 1991
Co. F1 Hybrid. Char. Two weeks earlier than Honey Dew. Slips from vine when ripe; thick, pale-green flesh, Sim. Honey Dew. Adapt. All but northernmost areas.

Meloso (Exp. desig. XPH 5097)-1985-Orig. and intr. by Asgrow Seed Co. F1 Hybrid. Char. Early maturing, Piel de Sapo type, with high initial fruit set. High soluble solids, excellent flavor. Sim. Piel de Sapo. Adapt. Spain.

Mission (Exp. desig. XPH 913) -1983-Orig. and intr. by Asgrow Seed Co. F1 Hybrid. Char. 5 to 6 days earlier than Topmark; round, medium-large, futureless fruit. Small cavity. Sim. Topmark. Adapt. southern Texas, southwestern desert areas of U.S.

Nova (Exp. desig. GSV 83-93)-1983-Orig. and intr. by Goldsmith Seeds, Inc. Char. Early, oblong to round-shaped coarse-netted hybrid muskmelon. Sutures shallow. Orange flesh with excel lent sugar readings. Res. PMR. Adapt. Texas.

Samson -Orig. and intr. by Arco Seed Co. F1 Hybrid. Sim. Topmark. Adapt. Southwestern U.S.

Sunrise (Exp. desig. XP 673)-1987-Orig. and intr. by Agway, Inc. F1 Hybrid. Char. 65-day maturity, good vine vigor; fruit heavily netted and moderately ribbed. Sim. Hybrid Earligold. Adapt. Norheastern U.S.

\section{OKRA}

Prelude (Exp. desig. NV 2555)-1984-Orig. and intr. by Northrup King Co. Open poll. Char. fluted pod matures in $\approx 48$ days; for fresh market and processing; pod medium dark green; seven to eight carpels, 3 1/2" in length. Sim. Clemson Spineless. Adapt. All okra-producing areas. PVP application pending.

\section{ONION}

Blitz-1988-Orig. and intr. by Asgrow Seed Co. F1 Hybrid. Char. Excellent storage quality. Res. Pink Root. Tol. Alternaria . Sim. Spartan Sleeper. Adapt. Long-day areas requiring early maturity.

Brilliant (Exp. desig. ARCO 3059W)-1987-Orig. and intr. by Arco Seed Co. F1 Hybrid. Char. Early with glossy white bulbs; more bolt-resistant than similar varieties. Res. Pink Root. Sim. Early Supreme. Adapt. Short-day areas.

Bullseye (Exp. desig. 219W9)-1985-Orig. and intr. by FerryMorse Seed Co. F1 Hybrid. Par. FM inbreds. Char. Spanish-type hybrid, highly single centered with thick rings. Globe-shaped with straw-colored skins. Res. Pink Root. Sim. CIMA. Adapt. New York, Wisconsin, Michigan.

Celebrity (Exp. desig. W 133)-1984-Orig. and intr. by Crookham Co. F1 Hybrid. Par. (P54-306prrxP-USSprrII) Cr110C. Char. A Yss hybrid for the Idaho and eastern Oregon area and other areas where full Spanish types are grown. Bulbs medium to high globe shape. Scales yellowish-brown and adhere well through storage. Res. Very Tol. to Idaho and eastern Oregon strains of Pink Root. Sim. Utah Sweet Spanish. Adapt. Areas where Yellow Sweet Spanish is grown.

Cimmaron (Exp. desig. ARCO 7063-4)-1986-Orig. and intr. by Arco Seed Co. F1 Hybrid. Char. Vigorous, high-yielding, uni form, attractive yellow onion with high frequency of single-tentered bulbs. Intermediate daylength response. Limited storage potential. Res. Good Tol. to Pink Root. Sim. New Mexico Grano. Adapt. Northern Texas, New Mexico, San Joaquin Valley of California.

Class Pak (Exp. desig. 231W9)-1985-Orig. and intr. by FerryMorse Seed Co. F 1 Hybrid. Par. FM Hybrids. Char. Late-season, long-storage class; bulbs light-medium bronze color, skins tight. Res. Pink Root. Sim. Surecrop. Adapt. New York, Michigan, Wisconsin, Ohio, Ontario.

Colossol PRR -1980-Orig. and intr. by Arco Seed Co. Open poll. Char. Large, yellow grano type. Res. Pink Root and bolting. Sim. Early White Supreme. Adapt. Southwestern U. S., Mexico. PVP Certificate No. 7900076. 
Contessa (Exp. desig. XP 3200)-1986-Orig. and intr. by Asgrow Seed Co. Open poll. Char. Early, uniform, short-day type wth large, white, deep-globed bulbs; small root plate. Res. Tol. Pink Root and bolting. Sim. Early White Supreme. Adapt. Southwestern U.S., Mexico. PVP Certificate No. 8500193.

Crystal Wax PRR - Orig. and intr. by Arco Seed Co. Open poll. Res. Pink Root. Sim. L303. Adapt. Short-day areas.

Crystal Wax Pickling PRR -1983-Orig. and intr. by Arco Seed Co. Open poll. Char. Very flat. Res. Pink Root. Sim. Crystal Wax. Adapt. Long-day pickling onion growing areas. PVP application pending.

Dessex Yellow PRR -1963-Orig. and intr. by Arco Seed Co. F1 Hybrid. Char. Early Yellow Bermuda-type onion. Res. Pink Root. Adapt. Short-day areas.

Diaga (Exp. desig. KFX999)-1984-Orig. and intr. by K-F Seeds. F1 Hybrid. Char. Early, White Granex type. Res. Pink Root. Sim. Robust. Adapt. Short-day growing areas.

Early Grand PRR -1981-Orig. and intr. by Arco Seed Co. Open poll. Res. Pink Root. Sim. Grano 502. Adapt. Short-day areas. PVP Certificate No. 800161.

Early Premium PRR -1965-Orig. and intr. by Arco Seed Co. F1 Hybrid. Char. Early, flattened globe yellow onion. Res. Pink Root. Adapt. Short-day regions.

Early Supreme PRR -1964-Orig. and intr. by Arco Seed Co. F1 Hybrid. Char. Early white hybrid. Res. Pink Root. Adapt. Shortday regions.

Glacier (Exp. desig. ARCO 6054)-1985-Orig. and intr. by Arco Seed Co. F1 Hybrid. Char. Bright white outer scales; less prone to greening than Southport White Globe; outstanding storage quality for a white onion. Sim. Blanco Duro. Adapt. Northern oniongrowing areas.

Glory PRR -1981-Orig. and intr. by Arco Seed Co. Open poll. Res. Pink Root. Adapt. Intermediate onion areas. PVP Certificate No. 8100128.

Gold Rush PRR -1983-Orig. and intr. by Arco Seed Co. F1 Hybrid. Char. Very early, uniform, yellow onion with little doubling. Adapt. Short-day regions.

Golden Yellow -1964-Orig. and intr. by Arco Seed Co. F1 Hybrid. Char. Good storage for a short-day onion. Adapt. Short-day regions.

Henry's Special PRR -1965-Orig. and intr. by Arco Seed Co. F1 Hybrid. Char. Yellow. Res. Pink Root. Adapt. Short-day regions.

Maya (Exp. desig. XPH 739)-1985-Orig. and intr. by Asgrow Seed Co. F1 Hybrid. Char. Midseason, jumbo, long-day Spanish hybrid, with excellent storage potential. Sim. Vega Adapt. Northwestern U.S. (Idaho, Oregon, Washington), Colorado, Central California, northern Texas.

Midstar (Exp. desig. ARCO 1607)-1986-Orig. and intr. by Arco Seed Co. F1 Hybrid. Char. Vigorous, uniform, white onion with high frequency of single-centered bulbs. Intermediate day length response. Limited storage potential. Res. Tol. Pink Root. Sim. New Mexico Grano. Adapt. Northern Texas, New Mexico, San Joaquin Valley of California.

Paradise PRR -1981-Orig. and intr. by Arco Seed Co. Open poll. Res. Pink Root. Adapt. Intermediate onion Areas. PVP Certificate No. 8100129.

Paragon (Exp. desig. DEXP 1317-1)-1986-Orig. and intr. by Arco Seed Co. F1 Hybrid. Char. Early maturity with excellent storage quality and very hard flesh. Uniform globe with medium brown scales that adhere well in storage. Sim. Downing Yellow Globe. Adapt. Northern onion-growing areas.

Primero PRR -1968-Orig. and intr. by Arco Seed Co. Open poll. Res. Pink Root. Adapt. Short-day onion areas.

Red Commander PRR -1971 - Orig. and intr. by Arco Seed Co. F1 Hybrid. Res. Pink Root. Adapt. Short-day onion areas.

Regal PRR -1980-Orig. and intr. by Arco Seed Co. Open poll. Char. Early with excellent color. Res. Pink Root. Adapt. Short-day onion areas. PVP Certificate No. 8100130.

Ringer Grano PRR - Orig. and intr. by Arco Seed Co. Open poll. Char. High single-centered Grano. Res. Pink Root. Adapt. Short-day areas.
Rip Van Winkle (Exp. desig. 227W2)-1985-Orig. and intr. by Ferry-Morse Seed Co. Par. FM inbreds. Char. Early maturity coupled with good storage qualities. Sim. Spartan Sleeper. Adapt. New York, Wisconsin, Michigan, Ontario, Quebec.

Robust White PRR -1964-Orig. and intr. by Arco Seed Co. F1 Hybrid. Char. White hybrid. Res. Pink Root. Adapt. Short-day onion regions.

Rojo (Exp. desig. ARCO 407R)-1987-Orig. and intr. by Arco Seed Co. F1 Hybrid. Char. Early, large, thick, flat bulbs with excellent exterior and interior color. Res. Pink Root. Sim. Red Granex. Adapt. Short-day onion areas.

Sassy Brassy (Exp. desig. 232003 )-1985-Orig. and intr. by Ferry-Morse Seed Co. Par. FM inbreds. Char. Late-season storage type, dark bronze; tight skins. Res. Pink Root, Tol. Fusarium. Sim. Spartan Banner. Adapt. Michigan, Wisconsin, New York, Ontario.

Special 38 -1983 - Orig. and intr. by Arco Seed Co. F1 Hybrid. Res. Pink Root. Sim. Henry's Special. Adapt. Short-day onion regions.

Sweet Amber (Exp. desig. XPH 77N76)-1986-Orig. and intr. by Crookman Co. F1 Hybrid. Char. Early mild Spanish hybrid with good single centers and thick, succulent rings; heavy amber scales that adhere well. Res. High level of tolerance to Pink Root. Sim. Yellow Sweet Spanish. Adapt. Areas where Yellow Sweet Spanish is grown.

Sweet Georgia (Exp. desig. KFX1100)-1984-Orig. and intr. by K-F Seeds. F1 Hybrid. Char. Early mild Granex type bolt-resistant. Res. Pink Root. Sim. F1 Hybrid Granex. Adapt. Short-day growing areas, especially Georgia.

Sweet Ring (Exp. desig. ARCO 92-3147Y)-1985-Orig. and intr. by Arco Seed Co. F1 Hybrid. Char. Matures 7 to 10 days earli er than Early Premium, highly single-centered, medium to jumbo globe-shaped bulbs. Res. Pink Root. Sim. Colossal. Adapt. Shortday growing areas.

Sweet Vidalia (Exp. desig. KFX-1133)-1985-Orig. and intr. by K-F Seeds. F1 Hybrid. Char. Bolt-resistant, high percent single centers. Unusual mildness when grown in Vidalia, Ga., area. Res. Pink Root. Sim. F1 Granex. Adapt. Short-day growing areas, especially Georgia.

Sweetex (Exp. desig. ARCO 532Y)-1987-Orig. and intr. by Arco Seed Co. F1 Hybrid. Char. Large, early Granex-type bulbs. Res. Pink Root. Adapt. Short-day onion areas.

Sweetheart (Exp. desig. 219W2)-1985-Orig. and intr. by Ferry-Morse Seed Co. F1 Hybrid. Par. FM inbreds. Char. Spanish type that can be spaced for jumbo sizes and will store slightly longer than most Spanish hybrids. Highly single-centered. Sim. CIMA. Adapt. Michigan, New York.

Tango (Exp. desig. Exp110-4)-1985-Orig. and intr. by Arco Seed Co. F1 Hybrid. Char. Dark red color extends to center of bulb when well-cured; bulbs uniform and globe-shaped with small necks and excellent storage qualities. Sim. Carmen. Adapt. Northern onion-growing areas.

Texspan PRR -1981-Orig. and intr. by Arco Seed Co. Open poll. Res. Pink Root. Sim. Ben Sheman. Adapt. Intermediate onion areas. PVP Certificate No. 800159.

Texstar 80 Yellow PRR -1980-Orig. and intr. by Arco Seed Co. F1 Hybrid. Res. Pink Root. Sim. Yellow Granex. Adapt. Shortday onion regions.

Tropicana Red PRR -1964-Orig. and intr. by Arco Seed Co. F1 Hybrid. Res. Pink root. Adapt. Short-day onion areas.

White Creoso PRR -1968-Orig. and intr. by Arco Seed Co. Open poll. Res. Pink Root. Adapt. Intermediate onion areas.

White Express -1982-Orig. and intr. by Arco Seed Co. F1 Hybrid. Res. Pink Root. Adapt. Short-day onion areas.

White Majesty PRR -1964-Orig. and intr. by Arco Seed Co. F1 Hybrid. Res. Pink Root. Adapt. Short-day onion areas.

White Tampico PRR -1982-Orig. and intr. by Arco Seed Co. F1 Hybrid. Res. Pink Root. Adapt. Short-day onion areas.

\section{PEA}

Bemol (Exp. desig. XP C1220)-1985-Orig. and intr. by AsHortSCIEnCE, Vol. 26(4), ApriL 1991 
grow Seed Co. Open poll. Char. Extremely small-sieve canner pea; medium maturity. Res. Fusarium Wilt, races 1 and 2; moderate res. Pea Leaf Roll. Sim. Kriter. Adapt. All pea areas. PVP Certificate No. 8400092.

Citadel (Exp. desig. XPF 157)-1986-Orig. and intr. by Asgrow Seed Co. Open poll. Char. Very small sieve freezer pea; medium maturity. Res. Fusarium Wilt, races 1 and 2. Sim. Dinos. Adapt. All pea areas. PVP application pending.

Elegance (Exp. desig. XPC 141)-1984-Orig. and intr. by Asgrow Seed Co. Open poll. Char. Small sieve canner pea; medium maturity afila plant type. Res. Fusarium Wilt, race 2. Sim. Mini. Adapt. All pea areas. PVP Certificate No. 84000104.

Envy (Exp. desig. XPC 156)-1985-Orig. and intr. by Asgrow Seed Co. Open poll. Char. Midseason canner pea with medium sieve size. Res. Fusarium Wilt, race 1; moderate res. to Powdery Mildew. Sim. EP 8221. Adapt. All pea areas. PVP Certificate No. 8400094.

Flair (Exp. desig. XPF 176)-1987-Orig. and intr. by Asgrow Seed Co. Open poll. Char. Small sieve, midseason, freezing pea. Res. Fusarium Wilt, race 1. Sim. Rally except it is a freezer. Adapt. Pea areas. PVP application pending.

Gain (Exp. desig. XPC 173)-1986-Orig. and intr. by Asgrow Seed Co. Open poll. Char. Medium-late afila canner type. Res. Fusarium Wilt, race 1; Mod. res. Powdery Mildew. Sim. Pomak. Adapt. Pea areas. PVP application pending.

Goya (Exp. desig. XPF 131)-1984-Orig. and intr. by Asgrow Seed Co. Open poll. Char. Fresh-market pea with large pods and medium color. Res. Fusarium Wilt, race 1; moderately resistant to Pea Leaf-Roll Virus. Sim. Rondo, but 4 days earlier. Adapt. Freshmarket pea areas. PVP application pending.

Jupiter (Exp. desig. C 82408)-1986-Orig. and intr. by Rogers Brothers Seed Co. Open poll. Char. Light-green berry with very small sieve size. Sim. Mini. Adapt. Generally adapted when specific diseases are not a problem. PVP application pending.

Mendota (Exp. desig. AVX 323-26) - Orig. and intr. by Sunseeds. Open poll. Char. A full-season determinate canner variety with Powdery Mildew resistance. Res. Powdery Mildew. Sim. Canners seed \#8221. Adapt. Midwestern and Northeastern U.S. PVP application pending.

Menuet (Exp. desig. XPC 134)-1984-Orig. and intr. by Asgrow Seed Co. Open poll. Char. Full-season, small sieve wrinkled canner pea. Res. Fusarium Wilt, races 1 and 2; moderate resistance to Pea Leaf-Roll Virus. Sim. Mini. Adapt. Pea canning areas.

Nomad (Exp. desig. Freezer 74115)-1986-Orig. and intr. by Rogers Brothers Seed Co. Char. Similar to Dark Skin Perfection, but pods are concentrated near the top $1 / 3$ to $1 / 4$ of the plant with some triple pods; about one sieve size smaller than DSP. Res. Fusarium Wilt, race 1. Sim. Dark Skin Perfection. Adapt. General. PVP application pending.

Oregon 523 (Exp. desig. OSU 523) -1985-Orig. and intr. by Oregon State Univ. Open poll. Char. adapted to commercial freez ing. Res. Pea Enation Mosaic and Fusarium Wilt, race 1. Sim. Small Sieve Freezer. Adapt. Pacific Northwest, esp. Willamette Valley of Oregon.

Payload (Exp. desig. XPC 135) -1985-Orig. and intr. by Asgrow Seed Co. Open poll. Char. Early large sieve canner pea with high yield. Res. Fusarium Wilt, race 1; Near Wilt, race 2. Sim. Dawn. Adapt. All pea areas. PVP Certificate No. 8400103.

Polaris (Exp. desig. AVX 329) - Orig. and intr. by Sunseeds. Open poll. Char. Early determinate mainseason freezer in the 4-5 sieve size. Sim. Mars. Adapt. Midwestern and Northeastern U.S. PVP application pending.

Rampart (Exp. desig. XPF 151)-1985-Orig. and intr. by Asgrow Seed Co. Open poll. Char. Late small sieve freezer pea with afila plant type. Res. Fusarium Wilt, race 1. Sim. Ivy. Adapt. All pea areas. PVP Certificate No. 8400107.

Saber (Exp. desig. XPC 136) -1985 - Orig. and intr. by Asgrow Seed Co. Open poll. Char. A late-maturing canner pea with small sieve size. Res. Fusarium Wilt, race 1; moderate resistance to Pea Leaf Roll. Sim. Nugget. Adapt. All canning pea areas.

Sunset (Exp. desig. AVX 326) - Orig. and intr. by Sunseeds. Open poll. Char. An early sweet canner with ability to set single, HortSCIEnCE, Vol. 26(4), ApriL 1991 double, and triple flowers and pods at the first and second fruiting nodes. Sim. Dane. Adapt. All pea growing areas. PVP Certificate No. 830074.

Treat (Exp. desig. XPF 175)-1987-Orig. and intr. by Asgrow Seed Co. Open poll. Char. Small sieve early to midseason freezer. Res. Fusarium Wilt, race 1. Sim. Trend. Adapt. Pea areas. PVP application pending.

Trek (Exp. desig. XPF 150)-1985-Orig. and intr. by Asgrow Seed Co. Open poll. Char. Afila plant; midseason, medium-sieve freezer. Res. Fusarium Wilt, race 1. Adapt. Wide. PVP application pending.

Utrillo (Exp. desig. XPF 149)-1985-Orig. and intr. by Asgrow Seed Co. Open poll. Char. Fresh-market pea with very large, darkgreen pods. Res. Fusarium Wilt, race 1; moderate resistance to Pea Leaf-Roll Virus. Sim. Rondo. Adapt. Wide. PVP application penaling.

Vulcan (Exp. desig. XPF 149)-1985-Orig. and intr. by Asgrow Seed Co. Open poll. Char. Large-sieve, medium maturity freezer pea with afila plant type. Res. Fusarium Wilt, race 1. Sim. Venus. Adapt. Wide. PVP Certificate No. 8400105.

\section{PEPPER}

Crispy Hybrid -1983-Orig. and intr. by W. Atlee Burpee Co. F1 Hybrid. Char. Medium-short plants that set medium-sized, blocky, thick-walled fruits over long period; green turning red at maturity. Sim. Bell Boy. Adapt. Wide.

Domino (Exp. desig. XPH 5106)—Orig. and intr. by Asgrow Seed Co. F1 Hybrid. Char. A long green/red thick-walled bell for greenhouse production. Res. TMV common strain. Sim. Lamuyo. Adapt. Italy, Spain, Southeastern U.S., Mexico.

Giant Ace - Orig. and intr. by Sunseeds. F1 Hybrid. Char. Abilit y to set a crop of fancy, four-lobed bell-shaped fruits. Res. Tobacco Mosaic Virus. Sim. Keystone Resistant Giant. Adapt. Wide.

Hidalgo -1985-Orig. and intr. by Arco Seed Co. Open poll. Char. Serrano type with larger and longer fruits than normal. Res. Tobacco Etch Virus, Tobacco Mosaic, Potato Y, Pepper Mottle. PVP application pending.

Prima Belle (Exp. desig. ARCO 3×29)-1987-Orig. and intr. by Sunseeds. Open poll. Char. Large, blocky four-lobed, bell-type with thick walls and medium dark-green color turning bright red at full maturity. Res. Tobacco Mosaic Virus. Sim. Emerald Giant. Adapt. California and some parts of Florida and Texas. PVP application pending.

Purple Belle (Exp. desig. P35)-1984-Orig. and intr. by Northrup King Co. F1 Hybrid. Char. Unique purple color when immature and red at maturity; Very early maturing with compact plant size; four-lobed, blocky fruit $41 / 2$ " $\times 4$ ". Res. TMV. Sim. Violetta. Adapt. Wide.

Tambell-2 -1985-Orig. and intr. by Arco Seed Co. Open poll. Char. Multiple virus-resistant. Res. Tobacco Mosaic, Tobacco Etch, Potato Y, and Pepper Mottle. Sim. Grande Rio 66. PVP application pending.

Wonder Giant - Orig. and intr. by Arco Seed Co. F1 Hybrid. Res. Tobacco Mosaic Virus. Sim. Cal Wonder type. Adapt. Wide.

Zippy Hybrid -1982-Orig. and intr. by W. Atlee Burpee Co. F1 Hybrid. Char. Mild-flavored hot pepper with long, narrow fruits; green turning red. Sim. Cayenne. Adapt. Wide.

\section{PUMPKIN}

Autumn Gold -1987-Orig. and intr. by Musser Seed Co., Inc. F1 Hybrid. Char. Early orange fruit color development permits culture in areas where other varieties may not mature. Adapt. Wide.

Bushkin (VP) -1985-Orig. and intr. by W. Atlee Burpee Co. Open poll. Char. Bush-type, naked-seeded golden-orange pumpkin -8 to $10 \mathrm{lb}$ with good flesh for pies and nearly hulless seeds for eating raw or roasted. Sim. Cinderella. Adapt. Wide. PVP Certificate No. 8400138. 


\section{RADISH}

Belle Glade (Exp. desig. HXP 3763)-1985-Orig. and intr. by Harris Moran Seed Co. Open poll. Char. A red globe radish, resistant to Fusarium Yellows and more tolerant to high or low temperatures than existing cultivars. Res. Fusarium Yellows. Sim. Fancy Red. Adapt. Florida. PVP application pending.

Crystal White (Exp. desig. HXP 2760)-1986-Orig. and intr. by Harris Moran Seed Co. Open poll. Char. White icicle type with persistent white shoulders; matures in 30 days. Res. Fusarium Yellows. Adapt. Wide. PVP application pending.

Dandy - Orig. and intr. by Sunseeds. Open poll. Char. Medium top, earlier than Red Prince K Strain, uniform color, globe shape. Res. Fusarium Yellows. Sim. Red Prince K Strain. Adapt. Florida, upper Midwestern U.S.

Easter Egg - 1983-Orig. and intr. by Alf Christianson Seed Co. F1 Hybrid (multi-line). Char. Combination of red, pink, purple, violet, and white radishes. Roots remain firm and crisp with good flavor. Maturity 28 days. Strap-leaved. Adapt. Wide.

Red Pak (Exp. desig. NV 3258)-1986-Orig. and intr. by Northrup King Co. Open poll. Char. Florida spring-type with high percentage of number ones. Res. Rhizoctonia and cracking. Sim. Red Prince and Red Devil B. Adapt. Florida (spring and fall). PVP application pending.

Snow Belle -1983-Orig. and intr. by Alf Christianson Seed Co. Open poll. Char. Round white radish with small tap root. Matures in 28 days. Remains firm for a long time with good flavor. Strap leaf top. Adapt. All areas. PVP Certificate No. 8200025.

\section{SPINACH}

Cascade (Exp. desig. Exp. Hybrid No. 58)—Orig. and intr. by Alf Christianson Seed Co. F1 Hybrid. Char. Medium-green smooth leaf. Petioles strong and growth erect. Fast-growing for winter season bunching or processing. Res. Tol. to Mildew (races 1, 2, and 3). Sim. Hybrid 424. Adapt. Wide.

Chinook II -1983-Orig. and intr. by Alf Christianson Seed Co. F1 Hybrid. Char. Fast-growing, medium-green, semi-savoy erect plant with strong petioles. Res. Tol to Mildew (races 1, 2, and 3). Sim. Early Hybrid No. 7. Adapt. Autumn planting.

Coho (Exp. desig. ACX 6)-1984-Orig. and intr. by Alf Christianson Seed Co. F1 Hybrid. Char. Upright, emerald dark green semi-savoy that is fast-growing and long-standing. Res. Tol. to Blue Mold 1, 2, and 3. Highly Tol. to White Rust. Sim. Melody. Adapt. Wide for fall and spring sowing.

Debut (Exp. desig. ARCO 202)-1985-Orig. and intr. by Arco Seed Co. F1 Hybrid. Char. Long-standing, dark-green, smooth-leaf type. Good for fresh market and processing. Res. Downy Mildew (races 1,2, and 3). Sim. Polka. Adapt. Western U.S. winter areas.

Green Valley II -1984-Orig. and intr. by Alf Christianson Seed Co. Open poll. Par. Selection out of Green Valley. Char. A semisavoy, medium, emerald glossy, green spinach for fall planting in White Rust problem areas. Res. Tolerant to Blue Mold 1 and 2. High tolerance to White Rust. Adapt. Texas, Arkansas, for fall planting where White Rust is a problem.

Olympia (Exp. desig. No. 144)-1982-Orig. and intr. by Alf Christianson Seed Co. F1 Hybrid. Char. Longstanding variety that is very fast growing. Smooth leaf and dark green. Res. Tol. Downy Mildew (races 1, 2, and 3). Adapt. Spring and summer sowing for bunching and processor.

Ozarka II- 1984-Orig. and intr. by Alf Christianson Seed Co. open poll. Par. Selection out of Ozarka. Char. A medium, curled green, savoy spinach for fall planting in White Rust problem areas. Res. Tol. to Blue Mold 1 and 2; high tolerance to White Rust. Sim. Ozarka, 612. Adapt. Texas, Arkansas, or where White Rust is a problem. For fall planting.

Rainier (Exp. desig. Exp. 4375)-1982-Orig. and intr. by Alf Christianson Seed Co. F1 Hybrid. Char. Fast growing and medium longstanding. Leaves smooth, dark green; growth is very erect. Fancy variety for bunching. Res. Tol. to Mildew (races 1, 2, and 3). Sim. Hybrid 424. Adapt. Wide for fall and spring bunching.
Sassy (Exp. desig. ARCO 77)-1986-Orig. and intr. by Arco Seed Co. F1 Hybrid. Char. Vigorous dark-green full savoy for fresh market and processing. Res. White Rust, Downy Mildew (races 1 and 2). Sim. Dixie Market. Adapt. Texas, Arkansas, Oklahoma, Southeastern U.S.

Skookum (Exp. desig. No. 157)-1982-Orig. and intr. by Alf Christianson Seed Co. F1 Hybrid. Char. Fast-growing longstanding semi-savoy with dark-green erect leaves. Res. Tol. to Blue Mold (races 1, 2, and 3). Mod. Tol. to White Rust. Sim. Hybrid Avon. Adapt. Wide.

Tyee (Exp. desig. No. 159)-1982-Orig. and intr. by Alf Christianson Seed Co. F1 Hybrid. Char. Very longstanding and fast-growing semi-savoy for market and home garden. Res. Tol. Downy Mildew 1 and 3. Sim. Melody. Adapt. Wide.

\section{SQUASH}

Baraka (Exp. desig. CHY 4021)—Orig. and intr. by Area Seed Co. Char. An early maturing, light-green, Lebanese-type squash. Plants medium in size with a semi-open bush and single stem. Fruit are 4" to 5" in length, smooth and slightly larger at the blossom end. Sim. Clarita. Adapt. Middle East countries.

Barq (Exp. desig. FMX 327D)-1985-Orig. and intr. by FerryMorse Seed Co. F1 Hybrid. Par. FM inbreds. Char. Vigorous, open plants with medium-light-green teardrop-shaped fruit. Sim. Victoria. Adapt. Middle East.

Blanquita (Exp. desig. ARCO 233) -1985-Orig. and intr. by Arco Seed Co. F1 Hybrid. Par. ARCO $195 \times$ ARCO 193. Char. Pale-green fruit with slight speckling. Sim. Clarita.

Blondie (Exp. desig. XPH 1435)-1986-Orig. and intr. by Asgrow Seed Co. F1 Hybrid. Char. Unique pale-yellow, long, cylindrical fruit open bush. Adapt. Wide.

Bushel -1984-Orig. and intr. by Hollar and Co., Inc. Open poll. Char. Lagenaria gourd, fruit spherical, diameter 12" to 18", green fruit weight to $100 \mathrm{lb}$. Sim. African Giant. Adapt. South.

Butter Blossom (Exp. desig. SQ 208)-1987-Orig. and intr. by Robson Seed Farms Co. Open poll. Char. Dark-green zucchini-type summer squash maturing in 45 days; high percentage of early male flowers. Sim. Corsair. Adapt. Wide.

Castle Black (Exp. desig. CHY 4008)-1983-Orig. and intr. by Arco Seed Co. F1 Hybrid. Char. Black zucchini type. Sim. Blackjack, Blackee.

Castlejade (Exp. desig. CHY 4005 ) —Orig. and intr. by Arco Seed Co. F1 Hybrid. Char. Long (7 1/4" to $81 / 2$ "), uniform, smooth, dark-green zucchini type with light-green flecks. Sim. Aristocrat, Zucchini Elite. Adapt. Wide.

Castle Pride (Exp. desig. CHY 4010)-1983-Orig. and intr. by Arco Seed Co. F1 Hybrid. Char. yellow crookneck hybrid. Sim. Dixie. Adapt. Wide.

Castle Queen (Exp. desig. CHY 4022)-1985-Orig. and intr. by Arco Seed Co. F1 Hybrid. Medium-dark-green zucchini with very slight flecking on open bushes. Sim. Ambassador, Seneca Zucchini. Adapt. Wide.

Consul (Exp. desig. XPH 1428)-1985-Orig. and intr. by Asgrow Seed Co. F1 Hybrid. Char. Early summer squash in the Senator/Seneca zucchini class. Sim. Senator. Adapt. Wide.

Corona (Exp. desig. XPH 1592)-1986-Orig. and intr. by Asgrow Seed Co. F1 Hybrid. Char. Pale-green/cream color with no green striping; Plant erect. Sim. Clarita. Adapt. Areas of Middle East using Clarita.

Early Acorn Hybrid -1982-Orig. and intr. by W. Atlee Burpee Co. F1 Hybrid. Char. Early maturing large acorn squash produced on short, semi-bush-type plants. Sim. Table Ace. Adapt. Wide.

Goldfinger (Exp. desig. XPH 1294)-1985-Orig. and intr. by Asgrow Seed Co. F1 Hybrid. Char. A yellow zucchini with firm seed cavity. Sim. Goldrush. Adapt. Wide.

Green Magic II -1986-Orig. and intr. by Musser Seed Co. F1 Hybrid. Char. Longer than Green Magic. Sim. Green Magic. Adapt. Wide.

Grisette (Exp. desig. CHY 4026)-1983-Orig. and intr. by Arco Seed Co. F1 Hybrid. Char. Medium grey-green fruit color with HortSCIENCE, Vol. 26(4), APrIL 1991 
some stripe. Sim. Caserta. Adapt. Middle East.

Jackpot (Exp. desig. 007041 )-1985-Orig. and intr. by Hollar and Co., Inc. F1 Hybrid. Char. Open bush, few spines, dark-green foliage, scalloped leaves. Sim. Black Beauty. Adapt. Wide.

Jade (Exp. desig. FMX 212)-1985-Orig. and intr. by FerryMorse Seed Co. F1 Hybrid. Par. FM inbreds. Char. Zucchini-type with medium green color, slight striping, and moderate speckling. Sim. Zucchini Elite. Adapt. Eastern U.S.

Lancer (Exp. desig. XPH 1546)-Orig. and intr. by Asgrow Seed Co. F1 Hybrid. Char. An early, long, cylindrical, light-colored, zucchini squash on a small, open bush. Sim. Senator/Seneca zucchini. Adapt. Wide.

Lemonbar (Exp. desig. XPH 1433)-1986-Orig. and intr. by Asgrow Seed Co. F1 Hybrid. Char. yellow straightneck summer squash. Sim. Lemondrop L. Adapt. Southeastern U.S.

Mellow Gold (Exp. desig. XP 931)-1987-Orig. and intr. by Agway Inc. F1 Hybrid. Char. Yellow, straightneck summer squash; Sim. Gold Slice. Adapt. Eastern U.S.

Midnight Express (Exp. desig. FMX 210)-1985-Orig. and intr. by Ferry-Morse Seed Co. F1 Hybrid. Par. FM inbreds. Char. Zucchini type with dark-green fruit, large open bush. Fruit slightly longer and slightly darker green than Onyx. Sim. Onyx. Adapt. West Coast.

Pavo (Exp. desig. XPH 1457)-1985-Orig. and intr. by Asgrow Seed Co. F1 Hybrid. Char. An early, smooth-skinned version of Dixie. Sim. Dixie. Adapt. Southeastern U.S.

Pie-N-Pie Hybrid -1985-Orig. and intr. by W. Atlee Burpee Co. F1 Hybrid. Char. Early golden crookneck hybrid with smooth wart-free skin, single stem, open plant habit. Sim. Sundance. Adapt. Wide.

Premier (Exp. desig. GSV 83-68)-1982-Orig. and intr. by Northrup King Co. F1 Hybrid. Char. An Elite-type summer squash, early medium-green glossy fruit color with open plant habit. Sim. Elite types. Adapt. Wide.

Rama (Exp. desig. XPH 1517)-1986-Orig. and intr. by Asgrow Seed Co. F1 Hybrid. Char. English Marrow-type summer squash; light green in color. Sim. Clarita. Adapt. Jordan, Syria, Saudi Arabia.

Regency (Exp. desig. GSV 83-71)-1983-Orig. and intr. by Northrup King Co. F1 Hybrid. Char. Open plant habit, nearly spineless summer squash with medium green, waxy appearance. Sim. Ambassador. Adapt. Wide.

Richgreen Hybrid Zucchini -1982-Orig. and intr. by W. Atlee Burpee Co. F1 Hybrid. Char. Compact, open, single-stem habit and very dark, glossy green cylindrical fruits. Sim. Ambassador. Adapt. Wide.

Rocky Gold -1983-Orig. and intr. by Hollar and Co., Inc. F1 Hybrid. Char. Zucchini-type fruit with shiny golden skin, palewhite flesh, no green color at either end of fruit. Sim. Burpee Golden Zucchini.

Shurouq (Exp. desig. FMX 109)-1985-Orig. and intr. by Ferry-Morse Seed Co. F1 Hybrid. Par. FM inbreds. Char. Vigorous bush type with early production of pale grey-green, smooth, blocky, tear-drop, marrow-type fruit. Sim. Victoria, Ghada. Adapt. Middle East.

Verdemax (Exp. desig. CHY 4007)—Orig. and intr. by Arco Seed Co. F1 Hybrid. Char. Dark-green zucchini-type squash borne on a medium-large, open, single-stemmed plant. Sim. Blackjack. Adapt. Wide.

Waltham Delite (Exp. desig. Waltham 8012)-1985-Orig. and intr. by Univ. of Massachusetts. Vendor: Arco Seed Co. F1 Hybrid. Char. Similar to Waltham Butternut, but 1 week earlier; slightly smaller-fruited with excellent storage characteristics. Res. Squash Borer. Sim. Waltham Butternut. Adapt. Northeastern U.S.

Zenith F1 -1985-Orig. and intr. by Hollar and Co. F1 Hybrid. Char. Uniform butternut-type fruit smaller than Waltham. Sim. Butternut Ponca.

\section{SWEET CORN}

Achiever (Exp. desig. AV 2524) — Orig. and intr. by Sunseeds. F1 Hybrid. Char. Sugary ( $\mathrm{su} / \mathrm{su}$ ) hybrid 81 -day relative maturity; HortScIEnce, Vol. 26(4), AprIL 1991 white silks, yellow kernels. 18- to 20-row whole kernel processor type. Adapt. Wide.

Arrestor - Orig. and intr. by Sunseeds. F1 Hybrid. Char. Sugary ( $\mathrm{su} / \mathrm{su}$ ) hybrid; 84-day relative maturity; white silks, yellow kernel processor type. Res. Races of Common Rust (Puccinia sorghi). Sim. Commander. Adapt. Midwestern and Northeastern U.S.

Bon Bon (Exp. desig. AV 2553)—Orig. and intr. by Sunseeds. F1 Hybrid. Char. Improved supersweet (sh2/sh2 su/su) 83-day relative maturity; white silk, light-yellow kernels: 14-18 rows. Sim. Florida Staysweet.

Calico Belle (Exp. desig. XPH 2639BC)-1987-Orig. and intr. by Asgrow Seed Co. F1 Hybrid. Char. High-quality midseason bicolor. Sim. Snowbelle. Adapt. Sweet corn areas with bicolor markets.

Carnival (Exp. desig. XPH 2451 BC)-1984-Orig. and intr. by Asgrow Seed Co. F1 Hybrid. Char. Full-season bicolor with good yield and quality, attractive appearance, for roadside or short-distance shipping. Res. Tol. to Stewart's Wilt and Smut. Sim. Sweet Sue. Adapt. All sweet corn areas.

Carousel (Exp. desig. XP111)-1985-Orig. and intr. by Agway Inc. Open poll. Char. 110- to 120-day open-pollinated ornamental corn producing 3"- to 5"-long ears in a wide range of colors. Ears average 14 to 16 rows with excellent tip fill and tip cover. Plants have two to three vigorous tillers and each tiller produces several marketable ears. Adapt. Eastern U.S.

Checkmate (Exp. desig. ARCO 5438)-1985-Orig. and intr. by Arco Seed Co. F1 Hybrid. Char. Bicolor, sugary shrunken, 76-day maturity. Sim. Agway 3611. Adapt. Northeastern, East Coast, Northern U.S.

Classic Touch (Exp. desig. Hybrid 80-2709)-1985-Orig. and intr. by Rogers Bros. Seed Co. F1 Hybrid. Char. Early bicolor hybrid with improved eating quality. Ears are 7" long with 12 to 14 rows of kernels. Sim. Sugar and Gold. Adapt. Canada and Northern U.S.

Cotton Candy EH (Exp. desig. 84-1491 EH)-1987-Orig. and intr. by Musser Seed Co. F1 Hybrid. Char. Early white cylindrical ear, good flags and husk protection, plant and husk reddish green color. Adapt. Wide.

Crystal Bell (Exp. desig. 83 WH 18)-1986-Orig. and intr. by Seedway, Inc. F1 Hybrid. Char. High row, excellent cold tolerance; high-quality, narrow, and deep kernels. Sim. Quicksilver. Adapt. Northeastern U.S., Great Lakes, Canada.

Early Arctic - Orig. and intr. by Sunseeds. F1 Hybrid. Char. Sugary (su/su) hybrid 63-day relative maturity; white silk, yellow kernels 12 to 14 rows. Extra early hybrid for home garden and market. Sim. Earliking. Adapt. Wide.

Even Sweeter (Exp. desig. XPH 2607W sh-2)-1987-Orig. and intr. by Asgrow Seed Co. F1 Hybrid. Char. Midseason white shrunken-2 hybrid. Sim. Snowbelle. Adapt. Wide.

Excellency (Exp. desig. FMX 162)-1985-Orig. and intr. by Ferry-Morse Seed Co. F1 Hybrid. Par. FM inbreds. Char. Full-season, rust-resistant hybrid. Ears cylindrical to tapered with good cover and flags, narrow kernels. Res. Rust. Sim. Stylepak. Adapt. Midwestern U.S.

FM 0502 (Exp. desig. E0502)-1985-Orig. and intr. by FerryMorse Seed Co. F1 Hybrid. Par. FM inbreds. Char. Stylepak processing type with good seedling vigor and high yield potential. Res. Head Smut, Bacterial Wilt, Tol. to Rust. Sim. Commander. Adapt. Northwestern, Midwestern, and Northeastern U.S.

Gold Ring (Exp. desig. NCX2029)-1985-Orig. and intr. by Harris Moran Seed Co. F1 Hybrid. Char. 80-day, midseason processing and fresh-market hybrid, 8 1/2" ear, 18-row count. Has performed well under rust pressure and environmental stress affecting fall corn crop. Res. Rust (some strains), Northern Corn Leaf Blight. Sim. Jubilee. Adapt. Wide.

Honey 'N Pearl (Exp. desig. SCH 4405)—Orig. and intr. by Illinois Foundation Seeds, Inc. F1 Hybrid. Char. Early shrunken-2 bicolor; sturdy plant type; Very large ear with excellent shape and straight kernel rows (16 to 18 rows); excellent eating quality. 76 days to harvest at Champaign, Ill. Res. Mod. res. to Rust, Stewart's Wilt, and Northern Corn Leaf Blight. Adapt. Wide.

Honey Gold -1985-Orig. and intr. by Henry Fields. F1 Hybrid. 
Par. $189 \times 110$. Res. Smut. Sim. Remarkable. Adapt. All corngrowing areas.

Illini Gold (Exp. desig. SCH 4057) — Orig. and intr. by Illinois Foundation Seeds, Inc. F1 Hybrid. Char. Mid-early maturity (79 days) yellow, shrunken-2 sweet corn with attractive kernel shape and good depth; single-stalk, sturdy plant type, tender and flavorful eating quality; Res. moderately resistant to Stewart's Bacterial Wilt. Adapt. Wide.

Ivory 'N Gold (Exp. desig. SCH 4401)—Orig. and intr. by Illinois Foundation Seeds, Inc. F1 Hybrid. Char. Early (75 days at Champaign, Ill.) shrunken-2 bicolor sweet corn with large ear and excellent eating quality; 14 to 16 kernel rows, with distinctive contrasting yellow and white kernel color. Res. Northern Corn Leaf Blight, mod. res. to Stewart's Wilt. Adapt. Wide.

Kodiak -Orig. and intr. by Sunseeds. F1 Hybrid. Char. Sugary (su/su) hybrid; 69-day relative maturity. Segregating light and dark silk color, yellow kernels: 12 to 14 rows. Sim. Golden Beauty. Adapt. Wide.

Napier (Exp. desig. GH 2681)-1982-Orig. and intr. by Rogers Brothers Seed Co. F1 Hybrid. Char. Full-season (85 days) yellow sweet corn for processing. Long ears with straight kernel rows and above-average eating quality. Suitable for canning and freezing; ineluding frozen cob corn. Sim. Commander. Adapt. Wide.

Rival (Exp. desig. XPH 2565)-1985-Orig. and intr. by Asgrow Seed Co. F1 Hybrid. Char. Early processing hybrid with very good quality. Res. Tol. to Smut. Sim. Reliance. Adapt. Wide.

Seneca Gold-N-Crystal (Exp. desig. RXB 8501)-1986-Orig. and intr. by Robson Seed Farms Co. F1 Hybrid. Char. Relative maturity (85 days); 16 to 18 rows, bicolored kernels, white silk, heterozygous, sugary enhancer gene. Res. Tol. to Stewart's Wilt. Sim. Sweet Sal. Adapt. Northern U. S., Canada.

Seneca Gold-N-Pearl (Exp. desig. RXB 6801)-1986-Orig. and intr. by Robson Seed Farms Co. F1 Hybrid. Char. Relative maturity 68 days, bicolored kernels, 12 to 14 rows with white silk, heterozygous sugary enhancer gene. Res. No tolerance to Stewart's Wilt. Sim. Sprite. Adapt. Northeastern U. S., Canada.

Seneca Surlite (Exp. desig. RXP 356)-1987-Orig. and intr. by Robson Seed Farms Co. F1 Hybrid. Char. 76-day, white silks, yellow kernels, 12 to 14 rows. Res. Tol. to Stewart's Wilt. Sim. Seneca Warrior 225. Adapt. Northern U.S., Canada.

Silver Bullet (Exp. desig. FMX 164)-1986-Orig. and intr. by Ferry-Morse Seed Co. F1 Hybrid. Char. Early white hybrid with maturity comparable to Goldcrest. Plants vigorous red-tassled. Ears cylindrical to slightly tapered, husks dark green with good tip cover and long green flag leaves. Sim. Spring White and Earliqueen. Adapt. U.S.

Snowbelle (Exp. desig. XPH 2562 W)-1985-Orig. and intr. by Asgrow Seed Co. F1 Hybrid. Sim. Silver Queen. Adapt. Wide.

Stars-N-Bars (Exp. desig. 81 H63 BG)-1986-Orig. and intr. by Seedway, Inc. F1 Hybrid. Char. Bi-color, early, large ear, excellent cold tolerance. Sim. Early Crystal-N-Gold. Adapt. Northeastern U.S., Great Lakes, Canada.

Sugar Crunch (Exp. desig. AV 2561) - Orig. and intr. by Sunseeds. F1 Hybrid. Char. Improved supersweet (sh2/sh2 su/su), 83day relative maturity, white silks 12 to 16 kernel rows. Deep yellow kernels. Well-filled ears. Tight husk cover and good flag leaves. Res. Tol. to Common Rust (Puccinia sorghi). Sim. Florida Staysweet.

Sugar Treat (Exp. desig. ARCO 5290)-1985-Orig. and intr. by Arco Seed Co. F1 Hybrid. Char. Sugary shrunken, 82 days midwest, better husk cover than Florida Staysweet. Res. Helminthosporium turcicum, races I and II. Sim. Florida Staysweet. Adapt. Southern U.S., Japan, Taiwan.

Sunbeam (Exp. desig. NCX 2024)-1985-Orig. and intr. by Harris Moran Seed Co. F1 Hybrid. Char. 82-day, yellow hybrid for processing and fresh market. Ears 8 1/2", 20-row count, good tip fill and quality. Refined kernel for excellent canned production. Sim. Stylepak. Adapt. Wide.

Sun Sweet (Exp. desig. XP071)-1986-Orig. and intr. by Agway Inc. F1 Hybrid. Char. 85-day yellow sweet corn with 7.5" to 8" ears and 16 rows of kernels. Heterozygous hybrid. Res. Stewart's Wilt. Adapt. Northeastern U.S.

352
Sweet Belle (Exp. desig. XPH 2575)-1985-Orig. and intr. by Asgrow Seed Co. F1 Hybrid. Char. An advanced shrunken-2 sweet corn adapted for shipping, local market, canning, and freezing. Sim. Florida Staysweet. Adapt. Wide.

Sweetchex (Exp. desig. AV 2523) - Orig. and intr. by Sunseeds. F1 Hybrid. Char. Sugary shrunken-2 (su/su sh2/Sh2) hybrid 82-day relative maturity. White silks, bicolor with good husk cover and flag leaves. Adapt. Wide.

Sweetie (Exp. desig. AV 2519)—Orig. and intr. by Sunseeds. F1 Hybrid. Char. Improved supersweet (sh2/sh2 su/su) hybrid in 82day relative maturity. White silks, 14 to 18 kernel rows, yellow kernels. Res. Tol. Common Rust (Puccinia sorghi).

Thermal (Exp. desig. XPH 2557)-1983-Orig. and intr. by Asgrow Seed Co. F1 Hybrid. Char. Midseason yellow shipper Sim. Comanche. Adapt. Southwestern U.S.

Twice-as-Nice (Exp. desig. XP 008)-1987-Orig. and intr. by Agway Inc. F1 Hybrid. Char. 80-day bicolor shrunken-2 (sh2) hybrid with 7.5 " to 8 " ears averaging 14 to 18 rows of kernels. Plants are $6^{\underline{a}}$ to $7^{\underline{a}}$ tall. Sim. Summer Sweet Brand Bicolor 7802. Adapt. Eastern U.S

White Knight (Exp. desig. XPH 2554 W)- 1984-Orig. and intr. by Asgrow Seed Co. F1 Hybrid. Char. White corn 4 days earlier than Silver Queen. Res. Tol. to Stewart's Wilt and Smut. Sim. Comet. Adapt. Wide.

Yankee Belle (Exp. desig. XPH 2559 sh 2)-1983-Orig. and intr. by Asgrow Seed Co. F1 Hybrid. Char. Medium-early yellow shrunken-2. Adapt. Northern U.S.

\section{TOMATO}

Alta (Exp. desig. CXD 100)-1985-Orig. and intr. by Campbell Soup Co. F1 Hybrid. Char. Firm midseason square, processing type with compact vine. Res. V1 and F1. Adapt. California.

Amigo (Exp. desig. GSV 83-06)-1983-Orig. and intr. by Northrup King Co. F1 Hybrid. Char. Semi-indeterminate vine adapted for pole culture. Fruits are medium to medium-large. Dark green shoulder, jointed. Res. Verticillium, Fusarium (race 1) Nematodes, TMV. Adapt. Mexico and Florida.

Baja (Exp. desig. GSV 84-52 )-1983-Orig. and intr. by Northrup King Co. F1 Hybrid. Char. Vigorous growth habit adapted for pole cultivation for early, medium-, and late-season plantings; large fruit with green shoulders. Res. Verticillium, Fusarium (races 1 and 2), TMV. Adapt. Mexico.

Basket King Hybrid -1982-Orig. and intr. by W. Atlee Burpee Co. F1 Hybrid. Char. Well-branched determinate vine; round fruits of good flavor. Sim. Presto. Adapt. Wide.

Burton (Exp. desig. ARCO 576)-1985-Orig. and intr. by Arco Seed Co. F1 Hybrid. Par. Hayslip is female parent. Char. Midseason, large determinate vine, globe-shaped fruit; green shoulders, normal joint, tight blossom scars. Res. Verticillium, Fusarium (races 1 and 2), Stemphyllium, Nematodes. Sim. Flor America. Adapt. Wide.

Carmelita (Exp. desig. Castlehy 1075)_-Orig. and intr. by Arco Seed Co. F1 Hybrid. Char. indeterminate, medium-sized, smooth fruit for trellis culture. Res. Verticillium, Fusarium (races 1 and 2), Nematodes, TMV. Sim. Carmelo. Adapt. San Diego, Mexico.

Casadvance (Exp. desig. Castlehy 1514)-1982-Orig. and intr. by A.L. Castle, Inc. F1 Hybrid. Char. Very early maturing processing type with good color and firmness. Res. Verticillium (race 1), Fusarium (races 1 and 2). Sim. Peto 31. Adapt. Semi-arid processing tomato-production areas.

Cascade (Exp. desig. CX 8106)-1984-Orig. and intr. by Campbell Soup Co. Open poll. Char. Firm, green shoulder, square, round; compact vine, mid-early processing type, with above-average solids. Res. V1 and F1. Sim. Fruit similar to VF6203, vine like UC82B. Adapt. California.

Castle 499 (Exp. desig. Castlex 499)-1978-Orig. and intr. by Arco Seed Co. Open poll. Char. Firm blocky processing type with higher soluble solids than UC 82. Res. Verticillium (race 1), Fusarium (race 1). Sim. UC 82. Adapt. Wide.

HortScience, Vol. 26(4), April 1991 
Castle Crown (Exp. desig. Castlehy 1061) - Orig. and intr. by Arco Seed Co. F1 Hybrid. Char. Smooth, jointless, deep globe shape, very firm fresh-market type. Res. Fusarium (races 1 and 2), Verticillium, Nematodes, Stemphyllium, Alternaria. Sim. Sunny. Adapt. California Mexico.

Castlehy 1017 -1978 - Orig. and intr. by A.L. Castle, Inc. F1 Hybrid. Char. High-yielding, high soluble solids processor type. Res. Verticillium (race 1), Fusarium (races 1 and 2). Sim. Castlemor F. Adapt. Semi-arid processing tomato growing areas.

Castlehy 1204 Improved -1978 - Orig. and intr. by A.L. Castle, Inc. F1 Hybrid. Char. Early maturing processing type with good color and firmness. Res. Verticillium (race 1), Fusarium (race 1). Sim. U.C. 105.

Castlejay (Exp. desig. Castlehy 1516)-1982-Orig. and intr. by A.L. Castle, Inc. F1 Hybrid. Char. Jointless (J2), uniform ripening (uu), whole-peel processor type. Res. Verticillium (race 1), Fusarium (races 1 and 2). Sim. Castle Red. Adapt. Wide.

Castle King (Exp. desig. Castlehy 1054) - Orig. and intr. by Arco Seed Co. F1 Hybrid. Char. Late-season fresh-market type; medium-size vine, large, firm fruit with concentrated set. Res. Verticillium, Fusarium (races 1 and 2), Stemphyllium. Sim. Royal Flush. Adapt. California central valley.

Castlemor Improved (Exp. desig. Castlemor C5)-1972-Orig. and intr. by A.L. Castle, Inc. Open poll. Char. Larger plant frame and higher yielding than VF 145B-7879 in San Joaquin Valley of California. Res. Verticillium (race 1), Fusarium (race 1). Sim. VF 145B-7879. Adapt. Semi-arid processing tomato-growing areas.

Castlepeel II (Exp. desig. Castlepeel II)-1982-Orig. and intr. by A.L. Castle, Inc. Open poll. Char. Compact vine, early maturing, round whole-peel type; easy peel character. Res. Verticillium (race 1), Fusarium (race 1). Sim. VF145 B-7879. Adapt. Semi-arid processing tomato-growing areas.

Castle Red (Exp. desig. Castlex 622)-1982-Orig. and intr. by A.L. Castle, Inc. Open poll. Char. Early jointless (j2) round wholepeel, uniform ripening type. Res. Verticillium (race 1), Fusarium (race 1). Sim. Murrieta. Adapt. Semi-arid processing tomato-growing areas. PVP Certificate No. 8200139.

Castlerock (Exp. desig. Castlex 489G)-1980-Orig. and intr. by A.L. Castle, Inc. Open poll. Char. Firm with high percentage of interlocular wall tissue, high color; high-viscosity processor type. Res. Verticillium (race 1), Fusarium (race 1). Sim. UC 82. Adapt.. Semi-arid areas. PVP Certificate No. 8100078.

Castleroyal (Exp. desig. 7W907C)-1983-Orig. and intr. by Arco Seed Co. Open poll. Char. Jointless (j2), uniform ripening deep blocky fruit. Res. Verticillium (race 1), Fusarium (races 1 and 2). Sim. UC 134. Adapt. Semi-arid processing tomato-growing areas.

Castlette (Exp. desig. Castlehy 1067)—Orig. and intr. by Arco Seed Co. Char. Firm jointless cherry tomato, medium determinate vine; about six to eight fruits/cluster. Res. Fusarium (race 1) and Verticillium. Sim. Cherry Grande. Adapt. Mexico, California.

Castlong UG [Exp. desig. (65-2 × 1339-12) Al]-1975-Orig. and intr. by A.L. Castle, Inc. Char. Early maturity, elongate fruit shape, uniform ripening processing type. Res. Verticillium (race 1), Fusarium (race 1). Sim. VF 65-2. Adapt. Semi-arid processing tomato-growing areas. PVP Certificate No. 8100077.

Centurion (Exp. desig. XPH 5101)-1986-Orig. and intr. by Asgrow Seed Co. F1 Hybrid. Char. Relatively firm-fruited, freshmarket type. Res. V, F1, F2, N, A, S, C. Sim. Nema 1200. Adapt. California.

Cherry Supreme (Exp. desig. $54 \times 221)$-Orig. and intr. by Arco Seed Co. Char. Large vine jointed cherry type. Res. Fusarium (race 1). Sim. Large Red Cherry. Adapt. San Diego, Mexico.

Cherry Sweet (Exp. desig. GSV 83-010)-1983-Orig. and intr. by Northrup King Co. F1 Hybrid. Char. Firm cherry type. Res. VFNT. Adapt. Wide.

Chunky (Exp. desig. AVX 7880)-Orig. and intr. by Sunseeds. F1 Hybrid. Char. Midseason square round. Res. V, (F1 \& F2). Adapt. Western and Southwestern U.S

Coronado (Exp. desig. Castlehy 1065)—Orig. and intr. by Arco Seed Co. F1 Hybrid. Char. Jointless fresh-market type with lightgreen shoulders. Res. Fusarium (races 1 and 2), Stemphyllium, AlHortScIENCE, Vol. 26(4), ApriL 1991 ternaria, Nematodes, Verticillium, TMV. Sim. Royal Flush. Adapt. Sari Diego, California coastal valleys.

Del Oro (Exp. desig. HYB. MOX 3076)-1984-Orig. and intr. by Harris Moran Seed Co. F1 Hybrid. Char. Early maturity, small stem scar, deep round fruit with thick walls for product use. Res. Verticillium, Fusarium (races 1 and 2), Nematodes, Alternaria. Adapt. California.

Diego (Exp. desig. MOX 3075)-1984-Orig. and intr. by Harris Moran Seed Co. Open poll. Char. Midseason compact plant, small stem scar, deep round fruit, thick walls for peeling and product use. Res. Verticillium, Fusarium (races 1 and 2), Alternaria. Adapt. California. PVP Certificate No. 8300172.

Eva (Exp. desig. AV 5715) - Orig. and intr. by Sunseeds. Open poll. Char. Midseason variety that performs well in high temperatures. Res. V, F. Sim. UC 82. Adapt. Wide.

Florabred (Exp. desig. Castlehy 1035)-1986-Orig. and intr. by Arco Seed Co. Char. Late, large vine for fresh market;large, firm jointless fruit. Res. Fusarium (races 1 and 2), Verticillium, Stemphyllium. Sim. Sunny. Adapt. Florida, Mexico.

Gator (Exp. desig. XPH 5011)-1986 - Orig. and intr. by Asgrow Seed Co. F1 Hybrid. Char. Large-fruited jointless hybrid. Res. V, F (1 \& 2), A, S, N. Sim. Duke. Adapt. Florida.

Genoa [Exp. desig. NVH 4759 (GSV-2401)]-1985-Orig. and intr. by Northrup King Co. F1 Hybrid. Char. Early jointed uniformripening (u) pear-shaped processing type with outstanding cover. Res. Verticillium and Fusarium (races 1 and 2). Sim. Castle Star, GS-31, Early Pear 1488. Adapt. California.

H300 (Exp. desig. MOX 3092)-1985-Orig. and intr. by Harris Moran Seed Co. F1 Hybrid. Char. Midseason processor variety with large, vigorous, determinate vine. Large globe fruit with good color and high solids. Res. Verticillium (race 1), Fusarium (races 1 and 2), Alternaria. Adapt. California.

Hermitage - Orig. and intr. by Sunseeds. F1 Hybrid. Char. Concentrated set; large smooth, firm, globe-shaped fruit. Res. Verticillium, Fusarium, TMV, Nematodes. Adapt. Southern U.S

Hybrid 724 (Exp. desig. XPH 724)-1984-Orig. and intr. by Asgrow Seed Co. F1 Hybrid. Char. Jointless hybrid for fresh market. Res. F1, F2, V1, Alternaria, Stemphylium. Sim. Duke. Adapt. U.S., Mexico, Australia.

Hybrid 896 (Exp. desig. XPH 896)-1984-Orig. and intr. by Asgrow Seed Co. F1 Hybrid. Par. Asgrow parents. Char. Early hybrid for peeling. Res. V1, F1. Sim. Pacesetter 694.

Hybrid 898 (Exp. desig. XPH 898) 1984-Orig. and intr. by Asgrow Seed Co. F1 Hybrid. Char. Midseason, product tomato with high solids and viscosity. Res. V1. F1, F2. Sim. Pacesetter 616. Adapt. Arid areas.

Hybrid 5040 (Exp. desig. XPH 5040)-1985-Orig. and intr. by Asgrow Seed Co. F1 Hybrid. Char. Pear-shaped processing whole peel type. Sim. Castlelong. Adapt. Italy and California.

Maranda (Exp. desig. XPH 5133)-1985-Orig. and intr. by Asgrow Seed Co. F1 Hybrid. Char. Marmande fruit shape. Sim. Marmande RAF. Adapt. Spain and Italy. PVP application pending.

Milano [Exp. desig. NVH 4757 (GSV4-2367)]-1985-Orig. and intr. by Northrup King Co. F1 Hybrid. Char. Early midseason, firm, pea-shaped processing type; jointed and uniform ripening for peeling; good internal color and vine cover. Res. Verticillium and $\mathrm{Fu}$ sarium (races 1 and 2). Sim. Castle Star, GS-31, Early Pear 1488. Adapt. California.

Missouri (Exp. desig. XP 4051)-1986-Orig. and intr. by Asgrow Seed Co. Open poll. Char. Large-fruited, jointless. Res. V, F. Sim. Rio Grande. Adapt. Italy. PVP application pending.

Mistic (Exp. desig. AVX 8384)-Orig. and intr. by Sunseeds. F1 Hybrid. Char. Midseason, jointless, fresh-market tomato with large, deep fruit. Res. V, F1 and F2. Sim. Sunny. Adapt. Florida, Mexico.

Mystro (Exp. desig. AVX 6002)—Orig. and intr. by Sunseeds. Open poll. Char. Early; blocky fruit for processing; peels well. Res. V, F. Adapt. Wide.

Oregon Spring (Exp. desig. OSU T65-5-1-14)—Orig. and intr. by Oregon State Univ. Open poll. Par. Severianin Starshot. Char. Early, large fruit; good seedless fruit set under cool temperature conditions of western Oregon. Res. Verticillium (strain 1). Sim. Se- 
verianin. Adapt. Western Oregon and Washington.

Pacific (Exp. desig. XPH 5074)-1986-Orig. and intr. by Asgrow Seed Co. F1 Hybrid. Char. Large-fruited fresh-market hybrid. Res. V, F1, F2, A, S. Sim. Contessa. Adapt. Western Mexico, Baja, California.

Propak (Exp. desig. XPH 5102)-1985-Orig. and intr. by Asgrow Seed Co. F1 Hybrid. Char. Firm, medium solids, processing hybrid. Res. V1, F1, F2, A, N. Sim. Centurion. Adapt. Middle East.

Ranch (Exp. desig. AV 5131) — Orig. and intr. by Sunseeds. Open poll. Char. Early-midseason, square-round for peeling. Res. V, F. Sim. UC 82. Adapt. Wide.

Reliant (Exp. desig. GSV 82-1131)-1983-Orig. and intr. by Northrup King Co. F1 Hybrid. Char. An early to midseason square, round processing hybrid with good solids and viscosity. Adapt. Western U.S.

Royal Delight (Exp. desig. Castlehy 1052)-1986-Orig. and intr. by Castle Seed Co. F1 Hybrid. Char. Medium-larg uniform fruit. Res. V, F, N, T. Sim. Celebrity, Ace. Adapt. U.S., Canada, Mexico.

Royal Star (Exp. desig. Arco 1514)-1986-Orig. and intr. by Arco Seed Co. F1 Hybrid. Char. Early; round fruit. Res. V, F1, F2.

Santa Clara (Exp. desig. XP 5200)-1986-Orig. and intr. by Asgrow Seed Co. Open poll. Char. Large, indeterminate; firm fruit. Res. V, F, S. Sim. Angela I-5100. Adapt. Brazil, Tropics.

Santiam (Exp. desig. OSU T65-9-2-13-9-1) - Orig. and intr. by Oregon State Univ. Open poll. Par. Severianin $\times$ Starshot. Char. Early and large-fruited; good seedless fruit set under cool temperature conditions in western Oregon. Res. Verticillium (strain 1). Sim. Severianin. Adapt. Western Oregon, Washington.

Single Beefsteak VFN -1983-Orig. and intr. by W. Atlee Burpee Co. Open poll. Char. Very large fruits with small blossom scar. Res. Verticillium, Fusarium, Nematodes. Sim. Super Steak Hybrid. Adapt. Wide. PVP Certificate No. 8200028.

Summit (Exp. desig. CXD 101)-1985-Orig. and intr. by Campbell Soup Co. F1 Hybrid. Char. Firm, round, midseason hybrid, high solids, compact vine. Res. V1, F1 and F2. Sim. UC 204C. Adapt. California.

Sundrop -1985-Orig. and intr. by W. Atlee Burpee Co. Open poll. Char. Globe-shaped, orange-fleshed fruits; indeterminate vine. Sim. Basket Pak for size but orange. Adapt. Wide.

Superior -(Exp. desig. $54 \times 224)-1981$ - Orig. and intr. by Arco Seed Co. F1 Hybrid. Char. Large vine, smooth fruit, Res. Verticillium, Fusarium (races 1 and 2), Nematodes, Stemphyllium. Sim. Casino Royale/Sunny. Adapt. San Diego.

Taylor (Exp. desig. ARCO 574)-1985-Orig. and intr. by Arco Seed Co. F1 Hybrid. Par. Florida 1A is female parent. Char. Midseason; deep oblate fruit with green shoulders; normal joint; very tight blossom scars. Res. Verticillium, Fusarium (races 1 and 2); Stemphyllium, Nematodes. Sim. Celebrity. Adapt. Florida, Southern California coast.

The Beef. (Exp. desig. Castlehy 1069)-1984-Orig. and intr. by Henry Field Seed and Nursery Co. F1 Hybrid. Char. A mediumearly extra-large, beefsteak-type fruit with smooth, fun, uniform ripening fruits. Res. Verticillium, Fusarium. Sim. Beefsteak.

Ural (Exp. desig. XPH 4029)-1986-Orig. and intr. by Asgrow Italia. F1 Hybrid. Char. Pear shape; vine requires little training. Sim. Hypeel 244. Adapt. Italy.

\section{WATERMELON}

Assal (Exp. desig. XPH 5082)-1985-Orig. and intr. by Asgrow Seed Co. F1 Hybrid. Char. An early maturing semi-round type, somewhat darker green than Chilean Black. About $17 \mathrm{lb}$. Very high fruit set. Sim. Chilean Black. Adapt. Wide.

Baronet (Exp. desig. XPH 962)-1984-Orig. and intr. by Asgrow Seed Co. F1 Hybrid. Char. Dark-green, cylindrical fruit, (20 to $24 \mathrm{lb}$ ) small-seeded, with concentrated set. Sim. Picnic in color. Adapt. Southwestern U.S., Mexico, Middle East.

Bush Baby II (Exp. desig. NVH 4274)-1985-Orig. and intr. by Northrup King Co. F1 Hybrid. Char. Bush type (3 to 5 feet across); round fruit with dark-green exterior; some green stripes. Fruit avg. 7 to $10 \mathrm{lb}$; earlier than Sugar Baby. Res. Fusarium. Sim. Sugar Baby. Adapt. Wide.

Cardinal (Exp. desig. XPH 5085)-1985-Orig. and intr. by Asgrow Seed Co. F1 Hybrid. Char. Early long/oval type with small seed with high seedling vigor. Sim. Cal Sweet. Adapt. Southwestern U.S., Italy.

Carmen F1 -1985-Orig. and intr. by Hollar and Co., Inc. F1 Hybrid. Char. Medium green exterior with dark gray/green stripes. Oval shape, medium size. Res. Fusarium Wilt. Adapt. Western, Midwestern, and Southern U.S.; Australia, Middle East.

Charleston Elite (Exp. desig. NV 4300) -1983-Orig. and intr. by Northrup King Co. Open poll. Char. Fruits are long cylindrical with blunt ends (avg. $24 \mathrm{lb}$ in weight). Seeds are brown mottled. Res. Fusarium Wilt and Anthracnose (race 1). Sim. Charleston Gray \#133 and Charleston Gray \#5. Adapt. Wide.

Crimson Tide (Exp. desig. NVH 4268)-1984-Orig. and intr. by Northrup King Co. F1 Hybrid. Char. Crimson Sweet class hybrid with a slightly darker stripe. Fruit (avg. $24 \mathrm{lb}$ ) semi-elongated with small, dark-brown seeds; matures in Crimson Sweet class. Res, Fusarium Wilt. Sim. Crimson Sweet. Adapt. Wide.

Delta Queen (Exp. desig. FMX 13)-1985-Orig. and intr. by Ferry-Morse Seed Co. F1 Hybrid. Char. Large, oblong, dark-green fruit with narrow black-green stripes. Sim. Chilean Black-Seeded types. Adapt. Wide.

Diamond King F1 -1985-Orig. and intr. by Hollar and Co., Inc. F1 Hybrid. Adapt. Midwestern and Southern U.S.

Huck Finn (Exp. desig. FMX 11)-1985-Orig. and intr. by Ferry-Morse Seed Co. F1 Hybrid. Par. FM inbreds. Char. Fruits large, blocky, oblong, with markings similar to Crimson Sweet. Sim. Crimson Sweet. Adapt. Wide.

Sangria (Exp. desig. NVH 4262)-1985-Orig. and intr. by Northrup King Co. F1 Hybrid. Char. Elongated dark-green fruit (avg. $23 \mathrm{lb}$ ) with a light stripe and small, black seeds. Matures in $\approx 87$ days. Res. Fusarium Wilt; Tol. Anthracnose. Sim. Allsweet and Calsweet. Adapt. Wide.

Sugarama (Exp. desig. NVH 4264)-1984-Orig. and intr. by Northrup King Co. F1 Hybrid. Char. Early Crimson Sweet-type fruit (avg. 16 lb). Sim. Crimson Sweet. Adapt. Wide.

Sundance (Exp. desig. XPH 957)-1985-Orig. and intr. by Asgrow Seed Co. F1 Hybrid. Char. Very early, round with rind color between Crimson Sweet and Chilean Black. For early harvest needs in southeastern U.S. Res. Fusarium Wilt race 1. Sim. Crimson Sweet. Adapt. Southeastern U.S.

Sunsweet (Exp. desig. NV 4301)-1984-Orig. and intr. by Northrup King Co. Open poll. Char. Early striped Allsweet type; with semi-elongate fruit (18 to $24 \mathrm{lb}$ ); medium-size dark-brown seeds; high in sugar. Res. Fusarium Wilt. Tol. Anthracnose. Sim. Allsweet and Calsweet. Adapt. Wide. PVP application pending.

Sweet Charlie (Exp. desig. NVH 4254)-1984-Orig. and intr. by Northrup King Co. F1 Hybrid. Char. Oblong Charleston Graytype for long-distance shipping. Matures 2 or 3 days earlier than Charleston Gray and produces fruit avg. $25 \mathrm{lb}$. Res. Fusarium Wilt. Sim. Charleston Gray. Adapt. Wide. 
VARIETY

24 Karat

Achiever

All Star

Alta

Alwaha

Amigo

Apache

Apex

Aragon

Argo

Arrestor

Assal

Atlantic

Atlantis

Aurora

Aurora

Autumn Gold

Baccus

Baja

Baraka

Baronet

Barq

Basket King Hybrid

Beauty

Belle Glade

Bemol

Beta III

Bix

Blanquita

Blazer

Blitz

Blondie

Bon Bon

Bonanza

Brasilia

Brilliant

Brock Imperial 84

Bronco

Buccaneer

Bullseye

Burpee Hybrid II

Burpeeana Hybrid II

Burton

Bush Baby II

Bushel

Bushkin (VP)

Butter Blossom

Calico Belle

Candid Charm

Cannon

Cardinal

Carmelita

Carmen F1

Carnival

Carolong

Caropak

Carousel

Casadvance

Cascade

Cascade

Castle 499

Castle Black

Castle Crown

Castle King

Castle Pride

Castle Queen
CROP

Carrot

Sweet Corn

Muskmelon

Tomato

Muskmelon

Tomato

Carrot

Broccoli

Muskmelon

Bean

Sweet Corn

Watermelon

Bean

Carrot

Cucumber

Muskmelon

Pumpkin

Broccoli

Tomato

Squash

Watermelon

Squash

Tomato

Cucumber

Radish

Pea

Carrot

Lettuce

Squash

Bean

Onion

Squash

Sweet Corn

Broccoli

Carrot

Onion

Asparagus

Bean

Muskmelon

Onion

Cucumber

Cucumber

Tomato

Watermelon

Squash

Pumpkin

Squash

Sweet Corn

Cauliflower

Lettuce

Watermelon

Tomato

Watermelon

Sweet Corn

Carrot

Carrot

Sweet Corn

Tomato

Spinach

Tomato

Tomato

Squash

Tomato

Tomato

Squash

Squash

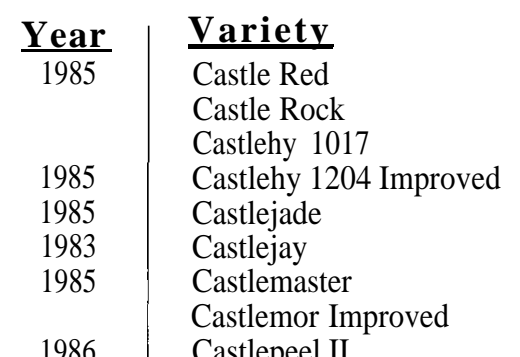

1986

1985

1985

1987

1985

1987

1986

1983

1984

1985

1982

1983

1985

1985

1987

1986

1985

1986

1988

1986

1982

1984

1987

1984

1986

1983

1985

1984

1984

1985

1985

1984

1985

1987

1987

1983

1986

1985

1985

1984

1988

1985

1985

1982

1984

1978

1983

1983

1985

Colossol PRR

Columbia

Comet A

Conqueror

Conquistador

Consul

Contessa

Corona

Coronado

Cortez

Cotton Candy EH

Crimson Tide

Crispy Hybrid

Crystal Bell

Crystal Wax PRR

Crystal Whhe

Cutlass

Dagger 78

Dandy

Debut

Del Oro

Delta

Delta Queen

Dessex Yellow PRR

Detroit Supreme

Diaga

Diamond King F1

Diego

Discovery
Crop

Tomato

Muskmelon

Tomato

Tomato

Squash

Tomato

Cucumber

Tomato

Tomato

Cucumber

Cucumber

Tomato

Cucumber

Tomato

Tomato

Tomato

Onion

Carrot

Carrot

Tomato

Muskmelon

Broccoli

Watermelon

Sweet Corn

Tomato

Tomato

Spinach

Tomato

Onion

Pea

Bean

Onion

Lettuce

Sweet Corn

Spinach

Onion

Commodore

Crystal Wax Pickling PRR

Muskmelon

Cucumber

Broccoli

Muskmelon

Muskmelon

Squash

Onion

Squash

Tomato

Onion

Onion

Radish

Bean

Carrot

Radish

Spinach

Tomato

Cucumber

Watermelon

Onion

Beet

Onion

Watermelon

Tomato

Carrot

Muskmelon 1985

Sweet Corn 1987

Watermelon 1984

Pepper 1983

Sweet Corn 1986

1983

1986

1985

1978

1985

1984

1986

1985 


\section{VARIETY}

Diyala

Domino

Dynasty

Eagle

Earligold F1

Earlimark F1

Early Acorn Hybrid

Early Arctic

Early Curly

Early Eastern Shipper

Early Glacier

Early Grand PRR

Early Premium PRR

Early Pride Hybrid

Early Prolific F-2

Early Snowflake

Early Supreme PRR

Easter Egg

Elegance

Envy

Eureka

Eva

Even Sweeter

Excellency

Explorer

FM 0502

Falcon

Fame

Fancipak

Flair

Florabred

Fremont

Gabilan

Gain

Galaxy

Gallena

Galo

Gator

Gemstone

Genesis

Genoa

Giant Ace

Gipsy

Glacier

Glacier

Gloria

Glory PRR

Gold Master

Gold Ring

Gold Rush PRR

Golden Harvest

Golden Pride

Golden Shipper

Golden Yellow

Goldfinger

Goya

Green Bouquet

Green Magic II

Green Towers

Green Valley II

Greenfield

Grisette

H300

Harvest Pride

Henry's Special PRR

Hermitage

Hidalgo

Honey 'N Pearl

Honey Gold

Honey Moon

Honeybush

356
CROP

Cucumber

Pepper

Cabbage

Carrot

Muskmelon

Muskmelon

Squash

Sweet Corn

Cabbage

Muskmelon

Cauliflower

Onion

Onion

Cucumber

Eggplant

Cauliflower

Onion

Radish

Pea

Pea

Bean

Tomato

Sweet Corn

Sweet Corn

Muskmelon

Sweet Corn

Carrot

Lettuce

Cucumber

Pea

Tomato

Cucumber

Leek

Pea

Broccoli

Carrot

Muskmelon

Tomato

Lettuce

Cabbage

Tomato

Pepper

Bean

Cauliflower

Onion

Cabbage

Onion

Muskmelon

Sweet Corn

Onion

Muskmelon

Muskmelon

Muskmelon

Onion

Squash

Pea

Basil

Squash

Lettuce

Spinach

Lettuce

Squash

Tomato

Muskmelon

Onion

Tomato

Pepper

Sweet Corn

Sweet Corn

Muskmelon

Muskmelon
YEA

1985

1985

1987

1985

1985

1982

1985

1987

1983

1981

1965

1983

1964

1983

1984

1985

1986

1987

1985

1983

1985

1987

1986

1985

1987

1986

1985

1984

1986

1984

1985

1985

1986

1981

1987

1985

1987

1983

1985

1984

1981

1987

1985

1983

1988

1988

1988

1964

1985

1984

1983

1986

1985

1984

1985

1983

1985

1986

1965

1985

1985

1987
VARIETY

Huck Finn

Huron

Hybrid 5040

Hybrid 724

Hybrid 896

Hybrid 898

Hybrid Aseel

Hystyle

Igloo

Illini Gold

Intrepid

Ivory 'N Gold

Jackpot

Jade

Jade

Javelin

Javelin 80

Jupiter

Kodiak

Kurinan

Lancer

Laureat

Lemonbar

Limelight Hybrid

Little Leprechaun

Longreen

Madrigal

Majestic

Manisa

Maranda

Mark II

Maya

Mellow Gold

Meloso

Mendota

Menuet

Midnight Express

Midstar

Mikado

Milano

Minia

Mission

Missouri

Mistic

Mystro

Nadine

Napier

Nomad

Nova

Ocala

Olympia

Olympian F1

Oregon 523

Oregon Spring

Ozarka II

Pacific

Paradise PRR

Paragon

Pavo

Payload

Pennant

Pie-N-Pie Hybrid

Podsquad

Polaris

Prelude

Premier

Prima Belle

Primepak

Primero PRR

Propak

Purple Belle

CROP

YEAR

Watermelon

1985

Carrot

1987

Tomato 1985

Tomato 1984

Tomato 1984 


\begin{tabular}{|c|c|c|c|c|c|}
\hline VARIETY & CROP & $\underline{\text { Year }}$ & VARIETY & CROP & $\underline{\text { Year }}$ \\
\hline Rainier & $\overline{\text { Spinach }}$ & $\overline{1982}$ & $\overline{\text { Sun Sweet }}$ & $\overline{\text { Sweet Corn }}$ & 1986 \\
\hline Rama & Squash & 1986 & Sunbeam & Sweet Corn & 1985 \\
\hline Rampart & Pea & 1985 & Sundance & Watermelon & 1985 \\
\hline Ranch & Tomato & & Sundrop & Tomato & 1985 \\
\hline Red Ace & Beet & 1981 & Sunrise & Muskmelon & 1987 \\
\hline Red Commander PRR & Onion & 1971 & Sunset & Pea & \\
\hline Red Pak & Radish & 1986 & Sunsweet & Watermelon & 1984 \\
\hline Regal PRR & Onion & 1980 & Superior & Tomato & 1981 \\
\hline Regency & Squash & 1983 & Sweet Amber & Onion & 1986 \\
\hline Reliant & Tomato & 1983 & Sweet Belle & Sweet Corn & 1985 \\
\hline Richgreen Hybrid Zucchini & Squash & 1982 & Sweet Charlie & Watermelon & 1984 \\
\hline Ringer Grano PRR & Onion & 1968 & Sweet Delight & Cucumber & 1983 \\
\hline Rip Van Winkle & Onion & 1985 & Sweet Georgia & Onion & 1984 \\
\hline Rival & Sweet Corn & 1985 & Sweet Ring & Onion & 1985 \\
\hline Robust White PRR & Onion & 1964 & Sweet Vidalia & Onion & 1985 \\
\hline Rock & Cucumber & 1983 & Sweetchex & Sweet Corn & \\
\hline Rocky Gold & Squash & 1983 & Sw e e t e $x$ & Onion & 1987 \\
\hline Rojo & Onion & 1987 & Sweetheart & Onion & 1985 \\
\hline Rosa & Lettuce & 1981 & Sweetie & Sweet Corn & \\
\hline Royal Delight & Tomato & 1986 & Sweetie B.S. & Lettuce & 1981 \\
\hline Royal Star & Tomato & 1986 & Tambell-2 & Pepper & 1985 \\
\hline Rubra & Lettuce & 1981 & Tango & Onion & 1985 \\
\hline Saber & Pea & 1985 & Taylor & Tomato & 1985 \\
\hline Salad Bibb & Lettuce & 1985 & Tess & Bean & 1985 \\
\hline Samson & Muskmelon & & Texspan PRR & Onion & 1981 \\
\hline Sangria & Watermelon & 1985 & Texstar 80 Yellow PRR & Onion & 1980 \\
\hline Santa Clara & Tomato & 1986 & The Beef & Tomato & 1984 \\
\hline Santiam & Tomato & & Thermal & Sweet Corn & 1983 \\
\hline Sassy & Spinach & 1986 & Thrive & Bean & 1987 \\
\hline Sassy Brassy & Onion & 1985 & Tigris & Cucumber & 1985 \\
\hline Savory & Carrot & 1986 & Toudo Hybrid & Carrot & 1985 \\
\hline Seminole & Carrot & 1985 & Treat & Pea & 1987 \\
\hline Seneca Gold-N-Crystal & Sweet Corn & 1986 & Trek & Pea & 1985 \\
\hline Seneca Gold-N-Pearl & Sweet Corn & 1986 & Tropicana Red PRR & Onion & 1964 \\
\hline Seneca Sunlite & Sweet Corn & 1987 & Twice-as-Nice & Sweet Corn & 1987 \\
\hline Sentry & Bean & 1986 & Two Seasons Hybrid & Cabbage & 1982 \\
\hline Show Boat & Cabbage & 1985 & Tyee & Spinach & 1982 \\
\hline Shurouq & Squash & 1985 & Ural & Tomato & 1986 \\
\hline Silver Bullet & Sweet Corn & 1986 & Utrillo & Pea & 1985 \\
\hline Single Beefsteak VFN & Tomato & 1983 & Vantage & Broccoli & \\
\hline Six Pence & Carrot & 1985 & Verdemax & Squash & \\
\hline Skookum & Spinach & 1982 & Vista & Bean & 1987 \\
\hline Snow Belle & Radish & 1983 & Volare & Bean & 1985 \\
\hline Snowball T-1 & Cauliflower & & Vulcan & Pea & 1985 \\
\hline Snowball T-2 & Cauliflower & & Waltham Delite & Squash & 1985 \\
\hline Snowball T-3 & Cauliflower & & White Creoso PRR & Onion & 1968 \\
\hline Snowball T4 & Cauliflower & & White Express & Onion & 1982 \\
\hline Snowbelle & Sweet Corn & 1985 & White Knight & Sweet Corn & 1984 \\
\hline Special 38 & Onion & 1983 & White Majesty PRR & Onion & 1964 \\
\hline Sprinter & Carrot & 1985 & White Tampico PRR & Onion & 1982 \\
\hline Sprout & Bean & 1986 & Wonder Giant & Pepper & \\
\hline Stars-N-Bars & Sweet Corn & 1986 & Yankee Belle & Sweet Corn & 1983 \\
\hline Sugar Crunch & Sweet Corn & & Zenith F1 & Squash & 1985 \\
\hline Sugar Treat & Sweet Corn & 1985 & Zippy Hybrid & Pepper & 1982 \\
\hline Sugarama & Watermelon & 1984 & & & \\
\hline Summit & Tomato & 1985 & & & \\
\hline
\end{tabular}

\section{Seed Producers Submitting Varieties for Naming on ASHS Vegetable Variety List XXIII}

Agway Inc., P.O. Box 4741, Syracuse, NY 13221

Alf Christianson Seed Co., P.O. Box 98, Mount Vernon, WA 98273

Arco Seed Co., 110 E. Ross Road, El Centro, CA 92243

Asgrow Seed Co., 7000 Portage Road, Kalamazoo, MI 49001

A.L. Castle, Inc., P.O. Box 877, Morgan Hill, CA 95023

Campbell Soup Co., Cirt, Route 1, Box 1314, Davis, CA 95616

Crookham Company, P.O. Box 520, Caldwell, ID 83606-0520

Dessert Seed Co., 110 E. Ross Avenue, El Centro, CA 92243

Ferry-Morse Seed Co.,P.O. Box 100, Mountain View, CA 94042

Goldsmith Seed, Inc., P.O. Box 1349, Gilroy, CA 95020

Harris Moran Seed Co., 3670 Buffalo Road, Rochester, NY 14624

HortScience, Vol. 26(4), April 1991
Illinois Foundation Seeds, Inc., P.O. Box 722, Champaign, IL 61820

Musser Seed Co. Inc., 301 4th Avenue, S., Twin Falls, ID 83303-1406

Northrup King Co., P.O. Box 1827, Gilroy, CA 95020

Oregon State University, Department of Horticulture, Corvallis, OR 97331

Robson Seed Farm Corporation, 1 Seneca Circle, Hall, NY 14463

Rogers Bros. Seed Co., P.O. Box 80, Idaho Falls, ID 83402

Seedway, Inc., Hall, NY 14463

Sunseeds, 9531 W. 78th Street, \#229, Eden Prairie, MN 55344

Sunseeds, 9870 Fairview Road, Hollister, CA 95023

W. Altee Burpee Co., 300 Park Avenue, Warminster, PA 18974

University of Wisconsin, Department of Horticulture, Madison, WI 53706 